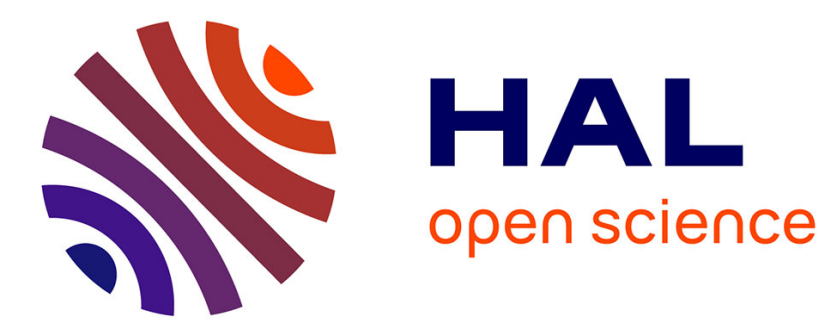

\title{
Estimates and bounds of dynamic permeability of granular media
}

Claude Boutin, Christian Geindreau

\section{To cite this version:}

Claude Boutin, Christian Geindreau. Estimates and bounds of dynamic permeability of granular media. Journal of the Acoustical Society of America, 2008, 124 (6), pp.3576-3593. hal-00941175

\section{HAL Id: hal-00941175 \\ https://hal.science/hal-00941175}

Submitted on 3 Feb 2014

HAL is a multi-disciplinary open access archive for the deposit and dissemination of scientific research documents, whether they are published or not. The documents may come from teaching and research institutions in France or abroad, or from public or private research centers.
L'archive ouverte pluridisciplinaire HAL, est destinée au dépôt et à la diffusion de documents scientifiques de niveau recherche, publiés ou non, émanant des établissements d'enseignement et de recherche français ou étrangers, des laboratoires publics ou privés. 


\title{
Estimates and bounds of dynamic permeability of granular media
}

\author{
Claude Boutin ${ }^{\text {a) }}$ \\ Laboratoire Geomateriaux, DGCB-URA CNRS 1652, Ecole Nationale des Travaux Publics de l'Etat, \\ Universite de Lyon, 69518 Vaulx-en-Velin Cedex, France \\ Christian Geindreau ${ }^{\text {b) }}$ \\ Laboratoire Sols-Solides-Structures-Risques, UMR 5521 CNRS-UJF-INPG, 38041 Grenoble Cedex 9, \\ France
}

This paper presents a study of dynamic permeability of porous media combining homogenization of periodic media (HPM) and the self-consistent method (SCM). By taking advantage of the physical principles identified with HPM, the application of SCM leads to the determination of two physically admissible dynamic permeability assessments, both different from that given by the cell model. A comparison with numerical modeling demonstrates the fairly good reliability of the three estimates for granular media consisting of a periodic array of spherical grains. Furthermore, the self-consistent values enable exact bounds for the dynamic permeability of a wide class of porous media to be derived with a clear identification of their microstructure (grain and fluid size distribution).

\section{INTRODUCTION}

Among the effective transfer coefficients of heat, mass, solute, etc., through porous media, the dynamic permeability is a physical parameter that is used in various domains of the mechanics, such as soil mechanics, petroleum engineering, geophysics, and acoustics of noise absorbing materials. The prediction of the permeability from the basic morphological information of a given medium is thus of interest both in scientific and industrial fields. It is worth noting that the dynamic permeability is a highly variable parameter (over several decades) according to pore size, solid volume fraction, and frequency. This makes its prediction even more important than other parameters and explains why its identification and characterization receive particular attention.

Two theoretical methods have made major contributions to the clarification of the nature of the dynamic permeability, namely, the pioneering phenomenological approach based on the thermodynamics - developed by Biot, ${ }^{1}$ continued, for instance, by Attenborough, ${ }^{2}$ Johnson et al. ${ }^{3}$ and Allard - and the micro-macro approach using the homogenization of periodic media (HPM) ${ }^{5-7}$ However, if the physics is well known, the question of reliable assessments and bounds of the dynamic permeability is still an open problem. As for static permeability, parametric studies were performed from numerical simulations ${ }^{8}$ or using the self-consistent method (SCM) as proposed by Berdichevsky and $\mathrm{Cai}^{9}$ for fibrous materials and by Boutin ${ }^{10}$ for granular media. Fibrous materials were also investigated by a cell model close to the SCM by Tarnow. ${ }^{11}$ In the dynamic range, exact expressions are available for plane slits or circular cylinders. As for more

\footnotetext{
${ }^{8)}$ Electronic mail: claude.boutin@entpe.fr

${ }^{b)}$ Electronic mail: christian.geindreau @ hmg.inpg.fr
}

realistic morphologies, Auriault et al. ${ }^{12}$ treated two dimensional channels, and Zhou and Sheng ${ }^{13}$ and Chapman and Higdon ${ }^{14}$ estimated, numerically, the dynamic permeability of packing of monodisperse spheres, for which Umnova et al. ${ }^{15}$ proposed an analytical calculation based on a cell model.

By combining the advantages of the HPM and SCM approaches, the aim of this paper is to go further in this modeling by establishing estimates and bounds of the dynamic permeability valid for porous media made of packing of polydisperse spheres. The HPM enables us (i) to derive rigorously the macroscopic law from the equations at the pore scale and (ii) to give the theoretical expression of the macroscopic coefficients - such as the dynamic permeability - whatever the periodic microstructure is. However the quantification of these coefficients requires numerical computations. This involves numerous simulations for identifying correlations between coefficients and microstructure. Complementary to HPM, the interest of SCM is to propose plausible values. They are based on analytical solutions in simplified configurations assumed to capture the salient morphologic features, i.e., in the present case, the connection of the fluid, the pore size, and the porosity. If the basic analytical solution is exact, the application of the result to real porous media is generally conjectured ${ }^{16}$ since, conversely to HPM, the microstructure is not treated explicitly. As for permeability, an additional difficulty arises because the governing equations are of different nature at the microscale (Navier-Stokes equation) and at the pore scale (Darcy law). Thus different estimates may be derived according to the assumptions taken for the basic solutions.

This paper is organized as follows. In Sec. II the derivation of the dynamic permeability using the HPM method is reviewed. Section III is devoted to the same problem using 
SCM and the principles identified by HPM. It is shown that two values of dynamic permeability can be conjectured, both being different from that given by the cell model. The interpretation of these results is covered in Sec. IV. First, a comparison with numerical modeling demonstrates the reliability of the estimates for granular media made of periodic arrays of identical spheres. Then, bounds of dynamic permeability are established for packing of polydisperse spheres.

\section{DERIVATION OF DYNAMIC PERMEABILITY BY PERIODIC HOMOGENIZATION}

\section{A. Basic principles of periodic homogenization}

The macroscopic representation of heterogeneous media makes sense only if there is a scale separation. This implies that $^{17}$

- the material is regular enough to be described by a representative volume (this is mathematically expressed by assuming a periodic material made of identical cells $\Omega$ of size) and

- the physical variables driving the phenomenon-for flow in porous media, the pressure at the leading order-varies according to a size $L$ larger than $l$.

To describe the variations at the well distinct lengths $L$ and $l$, two spatial variables are introduced, $\mathbf{x}$ for the macroscopic variations and $\mathbf{y}$ for the microscopic variations, $\mathbf{x}$ and $\mathbf{y}$ being related by the scale ratio,

$$
\varepsilon=l / L \ll 1, \quad \mathbf{y}=\varepsilon^{-1} \mathbf{x} .
$$

The small parameter $\varepsilon$ suggests seeking for any unknown $u$ (scalar, vector, etc.) in the form of asymptotic expansions in powers of $\varepsilon$. As the material periodicity and the scale separation induce the same periodicity for the physical quantities, all the terms are $\Omega$-periodic according to the variable $\mathbf{y}$,

$$
u(\mathbf{x}, \mathbf{y})=\sum_{0}^{\infty} \varepsilon^{i} u^{i}(\mathbf{x}, \mathbf{y}) \quad \text { with } \quad u^{i}(\mathbf{x}, \mathbf{y}) \quad \Omega \text {-periodic in } \mathbf{y} .
$$

The process of periodic homogenization consists in introducing the expansions in the two-variable rescaled equations that govern the physics at the local scale (i.e., equations where powers of $\varepsilon$ are used for expressing the order of magnitude of the dimensionless numbers and where the common spatial derivative is transformed into $\varepsilon^{-1} \partial_{y}+\partial_{x}$ ), then identifying the terms of the same power in $\varepsilon$, and finally solving the boundary value problems obtained in series.

\section{B. Dynamic permeability}

The derivation of the dynamic Darcy law from HPM was established by Levy ${ }^{18}$ and Auriault. ${ }^{5}$ The main results that will be used in the following are now recalled. For briefness, they are presented in the case of a rigid porous media saturated by an incompressible fluid. It is known that the same derivations apply in the case of elastic skeleton and compressible fluid. ${ }^{5}$ Consider a porous media (Fig. 1) of porosity $\phi$, saturated by a fluid of viscosity $\mu$ and density $\rho$,

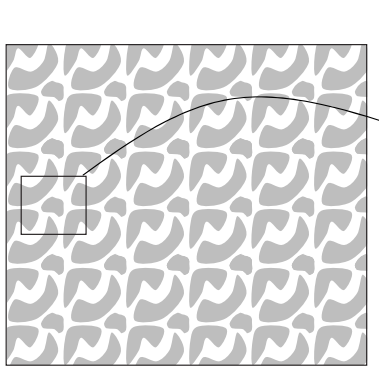

(a)

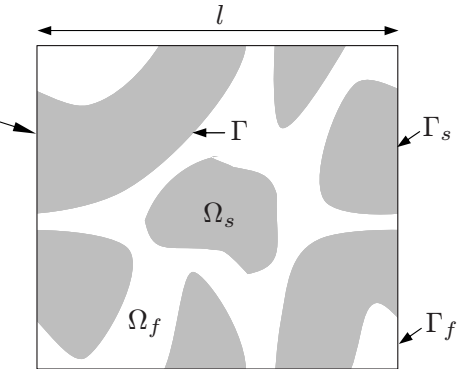

(b)
FIG. 1. (a) Macroscopic porous medium. (b) Periodic cell $\Omega$ of porous media. $\Omega_{f}$ and $\Omega_{s}$ are, respectively, the volume of the fluid and solid. $\Gamma$ is the fluid-solid interface inside $\Omega . \Gamma_{f}$ and $\Gamma_{s}$ are the fluid and solid interfaces at the boundary of $\Omega$. $\phi=\Omega_{f} / \Omega$ is the porosity.

submitted to small harmonic perturbations of frequency $f$ $=\omega / 2 \pi$. In the pores, the pressure variations $p$, fluid velocity $\mathbf{v}[\mathbf{D}(\mathbf{v})$ is the strain rate tensor] is driven by the following linearized equations, in which the term $e^{+i \omega t}$ is omitted.

- In the pores $\Omega_{f}$, the incompressibility [Eq. (2)], the viscous behavior [Eq. (3)], and the Navier-Stokes equation (momentum balance) [Eq. (4)] are written,

$$
\begin{aligned}
& \nabla \cdot \mathbf{v}=0, \\
& \boldsymbol{\sigma}=-p \mathbf{I}+2 \mu \mathbf{D}(\mathbf{v}), \\
& \nabla \cdot \boldsymbol{\sigma}=-\nabla p+\nabla \cdot[2 \mu \mathbf{D}(\mathbf{v})]=i \omega \rho \mathbf{v} .
\end{aligned}
$$

- On the fluid-solid interface $\Gamma$, the adherence condition is written as

$$
\mathbf{v}_{/ \Gamma}=\mathbf{0} \text {. }
$$

In these equations $\nabla, \nabla \cdot$, and $\Delta$ stand for the gradient, the divergence, and the Laplacian operators, respectively, and I is the unit tensor.

\section{Dimensional analysis and rescaling}

The physics of the flow has to be precisely stated. The dynamic Darcy regime is reached when the pressure gradient, viscous forces, and inertial forces are all of the same order of magnitude, i.e., when

$$
O(\nabla p)=O(\nabla \cdot[2 \mu \mathbf{D}(\mathbf{v})])=O(i \omega \rho \mathbf{v}) .
$$

The pores' geometry and the adherence condition enforce the velocity to vary at the pore scale. Therefore a macroscopic description is only possible if the pressure varies at a larger scale. This suggests expressing the pressure and the velocity as the products of reference pressure and velocity (subscript $r$ ), with dimensionless pressure and velocity (subscript $*$ ). These latter depend on both dimensionless variables $\mathbf{x} / \mathbf{L}$ $=\mathbf{x}^{*}$ and $\mathbf{x} / l=\mathbf{y}^{*}$, with $\mathbf{y}=\varepsilon^{-1} \mathbf{x}$,

$$
\begin{aligned}
p(\mathbf{x})= & p^{r} p^{*}\left(\mathbf{x}^{*}\right), \quad \mathbf{v}(\mathbf{x})=v^{r} \mathbf{v}^{*}\left(\mathbf{y}^{*}\right) \\
& \text { with } \quad p^{*}=O(1) ; \quad \mathbf{v}^{*}=O(1) .
\end{aligned}
$$

Thus, we have 


$$
\begin{aligned}
& O(\nabla p)=p^{r} / L, \quad O(\nabla \cdot[2 \mu \mathbf{D}(\mathbf{v})])=\mu v^{r} / l^{2}, \\
& O(i \omega \rho \mathbf{v})=\omega \rho v^{r},
\end{aligned}
$$

so that with Eq. (6), the viscous layer and the pore size are of the same order, i.e., $\left|\delta_{v}\right|=|\sqrt{\mu /(i \rho \omega)}|=O(l)$. Therefore, in a dimensionless form where all the terms are of the same order, the Navier-Stokes equation that properly accounts for the physics of the flow reads

$$
\begin{aligned}
& -\frac{p^{r}}{L} \nabla_{x^{*}}\left[p^{*}\left(\mathbf{x}^{*}\right)\right]+\frac{2 \mu v^{r}}{l^{2}} \nabla_{y^{*}} \cdot\left[\mathbf{D}_{y^{*}}\left(\mathbf{v}^{*}\left(\mathbf{y}^{*}\right)\right)\right] \\
& \quad=i \omega \rho v^{r} \mathbf{v}^{*}\left(\mathbf{y}^{*}\right)
\end{aligned}
$$

which may be rewritten, in terms of physical variables and derivatives, as follows:

$$
-\nabla_{x}[p(\mathbf{x})]+\nabla_{y} \cdot\left[2 \mu \mathbf{D}_{y}(\mathbf{v}(\mathbf{x}))\right]=i \omega \rho \mathbf{v}(\mathbf{x}),
$$

and changed into $x$-derivatives $\nabla_{y} \cdot\left[2 \mu \mathbf{D}_{y}\right]=\varepsilon^{2} \nabla_{x} \cdot\left[2 \mu \mathbf{D}_{x}\right]$, so that the viscous term in the Navier-Stokes equation (and thus in the stress) is rescaled by the factor $\varepsilon^{2}$. This leads Eqs. (3) and (4) to be modified to

$$
\begin{aligned}
& -\nabla p+\varepsilon^{2} \nabla \cdot[2 \mu \mathbf{D}(\mathbf{v})]=i \omega \rho \mathbf{v}, \\
& \boldsymbol{\sigma}=-p \mathbf{I}+\varepsilon^{2} 2 \mu \mathbf{D}(\mathbf{v}) .
\end{aligned}
$$

\section{Treatment by homogenization}

Homogenization is applied to Eqs. (2), (5), (8), and (9). First, momentum balance [Eq. (8)] at the order $\varepsilon^{-1}$ reduces to $-\nabla_{y} p^{0}=0$, giving, as expected, a constant pressure in the pores,

$$
p^{0}(\mathbf{x}, \mathbf{y})=P(\mathbf{x})
$$

Then, the velocity $\mathbf{v}^{0}$ and pressure $p^{1}$ are derived from equations $\left(8-\epsilon^{0}, 2-\epsilon^{-1}, 5-\varepsilon^{0}\right)$,

$$
S_{v}^{0}\left\{\begin{array}{c}
-\nabla_{y} p^{1}-\nabla_{x} P-i \omega \rho \mathbf{v}^{0}+\nabla_{y} \cdot\left[2 \mu \mathbf{D}_{y}\left(\mathbf{v}^{0}\right)\right]=0, \\
\nabla_{y} \cdot \mathbf{v}^{0}=0 \\
\mathbf{v}_{/ \Gamma}^{0}=0 \\
\mathbf{v}^{0} \text { and } p^{1} \text { are } \Omega-\text { periodic. }
\end{array}\right.
$$

This set of equations defines the linear dynamic permeability problem where $\nabla_{x} P$ acts as a forcing term. Consequently the solution has the form (Auriault ${ }^{5}$ )

$$
\mu \mathbf{v}^{0}(\mathbf{x}, \mathbf{y})=-\mathbf{k}^{i} \nabla_{x i} P, \quad p^{1}(\mathbf{x}, \mathbf{y})=\zeta^{i} \cdot \nabla_{x i} P+\widehat{p^{1}}(\mathbf{x}),
$$

where $\left(-\mathbf{k}^{i} / \mu, \zeta^{i}\right)$ are the three velocities and pressure distributions corresponding to the unit pressure gradient in the three directions, $\nabla_{x} P=\mathbf{e}_{i}$. They are complex and depend on the local spatial variables and frequency through the dimensionless variable $\mathbf{y} / \delta$. Finally macroscopic mass balance is derived from the compatibility condition, which must be satisfied by the local mass balance. By integrating $\left(2-\varepsilon^{0}\right)$ over the pore volume, we get

$$
\int_{\Omega_{f}}\left(\nabla_{y} \cdot \mathbf{v}^{1}+\nabla_{x} \cdot \mathbf{v}^{0}\right) d \Omega=0 .
$$

From the divergence theorem, the periodicity, and the adherence condition $\left(5-\varepsilon^{1}\right)$ on $\Gamma$, the first term vanishes. Then, inverting $\mathbf{y}$-integration and $\mathbf{x}$-differentiation, we have

$$
\nabla_{x} \cdot\left(\frac{1}{\Omega} \int_{\Omega_{f}} \mathbf{v}^{0} d \Omega\right)=\nabla_{x} \cdot \mathbf{V}=0,
$$

where $\mathbf{V}$ is the Darcy velocity. The macroscopic description reads

$$
\begin{aligned}
& \nabla_{x} \cdot \mathbf{V}=0, \quad \mathbf{V}=-\frac{1}{\mu} \mathbf{K} \nabla_{x} P, \\
& \mathbf{K}=\frac{1}{\Omega} \int_{\Omega_{f}} \mathbf{e}^{i} \otimes \mathbf{k}^{i} d \Omega=\mathbf{K}^{R}+i \mathbf{K}^{I},
\end{aligned}
$$

where $\mathbf{K}^{R}$ and $\mathbf{K}^{I}$ are the real and imaginary parts of the complex dynamic permeability tensor $\mathbf{K}$. The inverse form of the macroscopic description is written as

$$
\mu \mathbf{H} \cdot \mathbf{V}=-\nabla_{x} P, \quad \mathbf{H}=\mathbf{K}^{-1}=\mathbf{H}^{R}+i \mathbf{H}^{I} .
$$

The features of the tensor $\mathbf{H}$ derived by HPM $^{12}$ are written below in the isotropic case, i.e., when $\mathbf{H}=H \mathbf{I}$ (and $K=H^{-1}$ ).

- In the quasistatic regime (low frequency), i.e., when $l / \delta$ $\rightarrow 0$, viscous effects dominate the inertia. Consequently, we have

$$
H(\omega) \simeq \frac{1}{\mathcal{K}}+\frac{i \omega \rho}{\mu} \frac{\alpha_{0}}{\phi},
$$

where $\mathcal{K}$ is the intrinsic permeability and $\alpha_{0}$ is the low frequency tortuosity.

- At high frequencies, i.e., when $l / \delta \rightarrow \infty$, inertial effects dominate, and viscous effects are confined to a viscous layer, giving

$$
\frac{\mu}{i \omega \rho} H(\omega) \phi \simeq \alpha_{\infty}\left(1+\sqrt{\frac{M}{2} \frac{\omega_{c}}{i \omega}}\right),
$$

where $\alpha_{\infty}$ is the tortuosity, $M$ is a shape factor, and $\omega_{c}$ is the critical frequency delimiting the low and high frequency domains. This latter, which is obtained by equalizing viscous and inertial effects of the macroscopic flow, is written as

$$
\omega_{c}=\frac{\phi \mu}{\mathcal{K} \rho \alpha_{\infty}} .
$$

Following Johnson et al., ${ }^{3} \sqrt{(M / 2)\left(\omega_{c} / i \omega\right)}$ may also be expressed as $2 \delta / \Lambda$, where $\Lambda$ is the characteristic viscous length.

The dimensionless terms $K / \mathcal{K}$ and $H \phi \mu /\left(i \omega \rho \alpha_{\infty}\right)$ $=H \mathcal{K} \omega_{c} / \omega$ depend only on the dimensionless frequency $\omega / \omega_{c}$. Note that the complex dynamic density defined by

$$
\rho_{d}(\omega) i \omega \mathbf{V}=-\nabla_{x} P
$$

is related to $H(\omega)$ by $\rho_{d}(\omega)=\phi(\mu H / i \omega)$. 


\section{Variational formulation and energy consistency}

The variational formulation equivalent to the set $S_{v}^{0}$ is derived ${ }^{5}$ by taking the scalar product of the momentum balance with any field $\mathbf{w}$ belonging to the vector space $\mathcal{W}$ defined as

$$
\mathcal{W}=\left\{\mathbf{w} / \quad \mathbf{w} \quad \Omega \text {-periodic }, \quad \mathbf{w}_{/ \Gamma}=0, \quad \nabla \cdot \mathbf{w}=0\right\} .
$$

After integrating over the pore and denoting the average over the representative volume $|\Omega|^{-1} \int_{\Omega_{f}}-d \Omega$ by $\langle-\rangle$, one obtains

$$
\begin{aligned}
- & \left\langle\nabla_{y} p^{1} \cdot \mathbf{w}\right\rangle+\left\langle\nabla_{y} \cdot\left[2 \mu \mathbf{D}_{y}\left(\mathbf{v}^{0}\right)\right] \cdot \mathbf{w}\right\rangle-i \omega \rho\left\langle\mathbf{v}^{0} \cdot \mathbf{w}\right\rangle \\
& =\nabla_{x} P \cdot(\mathbf{w}) .
\end{aligned}
$$

From the divergence theorem, the first term may be transformed as

$$
-\left\langle\nabla_{y} p^{1} \cdot \mathbf{w}\right\rangle=\left\langle p^{1} \nabla_{y} \cdot \mathbf{w}\right\rangle-\frac{1}{\Omega} \int_{\partial \Omega_{f}} p^{1} \mathbf{w} \cdot \mathbf{n} d s=0
$$

because of the zero divergence, the adherence, and the periodicity of $\mathbf{w}$. Similarly, from the divergence theorem, we get

$$
\begin{aligned}
\left\langle\nabla_{y} \cdot\left[2 \mu \mathbf{D}_{y}\left(\mathbf{v}^{0}\right)\right] \cdot \mathbf{w}\right\rangle= & -2 \mu\left\langle\mathbf{D}_{y}\left(\mathbf{v}^{0}\right): \mathbf{D}_{y}(\mathbf{w})\right\rangle \\
& +\frac{1}{\Omega} \int_{\partial \Omega_{f}}\left[2 \mu \mathbf{D}_{y}\left(\mathbf{v}^{0}\right) \mathbf{n}\right] \cdot \mathbf{w} d s .
\end{aligned}
$$

The surface integral vanishes because of the adherence and periodicity. Thus, this results in

$$
\begin{aligned}
& \forall \mathbf{w} \in \mathcal{W}, \\
& 2 \mu\left\langle\mathbf{D}_{y}\left(\mathbf{v}^{0}\right): \mathbf{D}_{y}(\mathbf{w})\right\rangle+i \omega \rho\left\langle\mathbf{v}^{0} \cdot \mathbf{w}\right\rangle=-\nabla_{x} P \cdot\langle\mathbf{w}\rangle .
\end{aligned}
$$

Taking $\mathbf{w}=\overline{\mathbf{v}^{0}}$ as a field test (the overbar stands for conjugate) gives the following identity:

$$
\begin{aligned}
& 2 \mu\left\langle\mathbf{D}_{y}\left(\mathbf{v}^{0}\right): \mathbf{D}_{y}\left(\overline{\mathbf{v}^{0}}\right)\right\rangle+i \omega \rho\left\langle\mathbf{v}^{0} \cdot \overline{\mathbf{v}^{0}}\right\rangle=-\nabla_{x} P \cdot\left\langle\overline{\mathbf{v}^{0}}\right\rangle \\
& \quad=\frac{1}{\mu} \nabla_{x} P \cdot \overline{\mathbf{K}} \cdot \overline{\nabla_{x} P}=\mu \mathbf{V} \cdot \mathbf{H} \cdot \overline{\mathbf{v}} .
\end{aligned}
$$

The above relation demonstrates consistency of viscous and kinetic energies between the microscopic (right hand side) and macroscopic (left hand side) descriptions. Applied to fields $\mathbf{k}^{i}$ and $\mathbf{k}^{j}$, relation (15) enables proof of the symmetry and positiveness of $\mathbf{H}$ and $\mathbf{K}$, which read in the isotropic case $^{5}$ as

$$
K^{R}>0, \quad K^{I}<0 \quad \text { and } \quad H^{R}>0, \quad H^{I}>0 .
$$

The real and imaginary parts of $K / \mu$ (or $\mu H$ ) correspond to the viscous dissipation and the effective kinetic power produced by a cycle under a unit macroscopic pressure gradient in the direction $\mathbf{e}_{i}$ (or a unit macroscopic flow in the same direction). Concerning the variation of $H$ versus frequency, it may also be demonstrated (see Appendix A) that with a time dependence $e^{+i \omega t}$,

$$
\frac{d H^{R}}{d \omega}>0 \quad \text { and } \quad \frac{d\left(H^{I} / \omega\right)}{d \omega}<0 .
$$

Reformulated in terms of dynamic density [Eq. (14)], these inequalities become

$$
\frac{d \rho_{d}^{R}}{d \omega}<0 \quad \text { and } \quad \frac{d\left(\omega \rho_{d}^{I}\right)}{d \omega}<0 .
$$

\section{Links between the microscopic to macroscopic descriptions}

The quantities appearing in the macroscopic description are related to the following local quantities:

- The macroscopic velocity, $\mathbf{V}$, is the average of the local velocity.

- The Darcy pressure $P$ is the stress of zero order in the pores. Because of the periodicity, the stresses of higher order are self-equilibrated on the boundary of the cell, i.e.,

$$
\int_{\Gamma_{f}} \boldsymbol{\sigma}^{1} \mathbf{n} d s=0 .
$$

- The permeability tensor $\mathbf{K}$ expresses the energy consistency: the viscous dissipation and the kinetic powers developed within the representative cell and in the same volume of the equivalent Darcy media are identical.

In the next section, the information gathered from HPM is introduced in a self-consistent approach.

\section{SELF-CONSISTENT ASSESSMENT OF DYNAMIC PERMEABILITY}

The self-consistent approach enables a conjecture of the effective coefficients of heterogeneous media. ${ }^{19}$ The method consists in

- assuming the nature of the macroscopic behavior and considering a generic inclusion representative of the local physics in the medium,

- solving the basic problem in the inclusion submitted to a homogeneous macroscopic forcing term and expressing the energy equivalence between the inclusion and the equivalent medium, and

- deducing from this relation the macroscopic coefficients.

The SCM was applied to the static intrinsic permeability of fibrous ${ }^{9}$ and granular materials ${ }^{10}$. Tarnow ${ }^{11}$ and Umnova et al. ${ }^{15}$ used a similar philosophy but introduced a condition of vanishing vorticity at the cell boundary as an alternative to energy consistency.

\section{A. Problem definition}

Following the HPM results, the behavior of the equivalent macroscopic medium complies with the dynamic Darcy law described by Eq. (52). For convenience, the analysis is presented for isotropic media (the same reasoning also applies for the principal values of a macroscopic anisotropic media). The idea is to fit the inclusion with the same volume of equivalent media-whose $K$ is to be determined-when both are subjected to a uniform forcing pressure gradient $\mathbf{G}$ of amplitude $G$ and direction $\mathbf{E}$ (capital letters are used for the Darcy medium variables), 


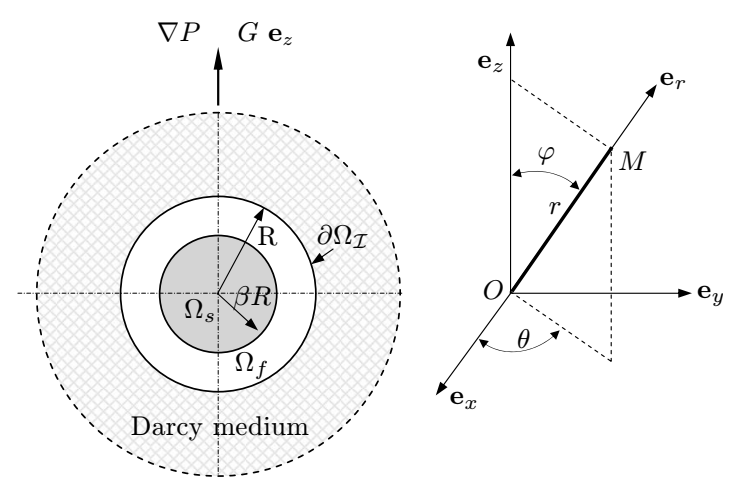

FIG. 2. The generic fluid-solid spherical inclusion $I$ of volume $\Omega_{i}$, boundary $\partial \Omega_{\mathcal{I}}$, and the associated spherical system of coordinates. Solid sphere: radius $R_{i}=\beta R$ and volume $\Omega_{s}$. Concentric spherical shell filled by the viscous Newtonian fluid: external radius $R$ and volume $\Omega_{f}=\Omega_{\mathcal{I}^{-}} \Omega_{s^{*}} . \phi=\Omega_{f} / \Omega_{\mathcal{I}}=1$ $-\beta^{3}$ is the porosity.

$$
\nabla P=\mathbf{G}=G \mathbf{E} \quad \text { so that } \quad \mathbf{V}=-\frac{K}{\mu} G \mathbf{E} .
$$

As for the generic inclusion $\mathcal{I}$ (of volume $\Omega_{\mathcal{I}}$ and surface $\partial \Omega_{\mathcal{I}}$ ), the simplest way to account for the connection of the fluid, the pore size, and the porosity is to consider a solid rigid sphere of radius $R_{i}=\beta R(0<\beta<1)$ and volume $\Omega_{s}$, surrounded by a concentric spherical shell filled by the fluid whose external radius is $R$ and whose volume is $\Omega_{f}=\Omega_{\mathcal{I}}$ $-\Omega_{s}$, so that the porosity $\phi=1-\beta^{3}$ (Fig. 2). Because of the spherical symmetry and the privileged direction introduced by the pressure gradient, the spherical coordinates $(r, \theta, \varphi)$ oriented as described in Fig. 2 will be used ( $\theta=0$ corresponds to $\mathbf{e}_{\mathbf{r}}=\mathbf{e}_{\mathrm{z}}=\mathbf{E}$ ).

The Navier-Stokes equation in the fluid shell, $\beta R<r$ $<R$, expressed with a single spatial variable, reads (lowercase letters are used for the variables of the fluid in the inclusion) as

$$
\begin{aligned}
& -\nabla p-\frac{1}{\delta^{2}} \mu \mathbf{v}+\Delta(\mu \mathbf{v})=\mathbf{0}, \\
& \nabla \cdot \mathbf{v}=0, \\
& p=\pi+\mathbf{r} \cdot \mathbf{G},
\end{aligned}
$$

where $\delta$ is the complex number $\delta=\sqrt{\mu / i \rho \omega}$ and $\mathbf{r}=r \mathbf{e}_{r}$. Note that the total pressure $p$ is decomposed into the pressure induced by the macroscopic uniform gradient, $\mathbf{r} \cdot \mathbf{G}$, and an additional pressure $\pi$. When adding the adherence condition on the solid, i.e., $\mathbf{v}(\beta R)=0$, the equations governing the fluid within the inclusion are identical to those derived by HPM, except for the periodicity (see II B 2).

\section{General expression of the fields $p$ and $v$}

To satisfy spatial isotropy, the fields in the inclusion must be isotropic functions of both position vector and forcing vector, namely, $\{\mathbf{r}, \mathbf{G}\}$. According the tensor theory, ${ }^{20}$ the pressure $p$ and the velocity $\mathbf{v}$ are written as

$$
p=\mathcal{F}_{p}(\mathbf{r} \cdot \mathbf{r}, \mathbf{G} \cdot \mathbf{G}, \mathbf{r} \cdot \mathbf{G}),
$$

$$
\mu \mathbf{v}=\mathbf{r} \mathcal{F}_{r}(\mathbf{r} \cdot \mathbf{r}, \mathbf{G} \cdot \mathbf{G}, \mathbf{r} \cdot \mathbf{G})+\mathbf{G} \mathcal{F}_{G}(\mathbf{r} \cdot \mathbf{r}, \mathbf{G} \cdot \mathbf{G}, \mathbf{r} \cdot \mathbf{G}),
$$

where $\mathcal{F}_{p}, \mathcal{F}_{r}$, and $\mathcal{F}_{G}$ are any functions of the three scalar $\mathbf{r} \cdot \mathbf{r}, \mathbf{G} \cdot \mathbf{G}$, and $\mathbf{r} \cdot \mathbf{G}$. In the present case, the solution depends linearly on G. Consequently, the general form of the solution can be specified more precisely through three independent functions of $r$,

$$
p=(\mathbf{r} \cdot \mathbf{G}) h_{1}(r), \quad \mu \mathbf{v}=\mathbf{r}(\mathbf{r} \cdot \mathbf{G}) f_{1}(r)+\mathbf{G} g_{1}(r) .
$$

Following Howells, ${ }^{21}$ these equations may be transformed conveniently into

$$
p=\mathbf{G} \cdot \nabla h(r), \quad \mu \mathbf{v}=\mathbf{G} \cdot[\nabla \otimes \nabla f(r)+g(r) \mathbf{I}] .
$$

The functions $f, g$, and $h$ have to be determined now to satisfy the Navier-Stokes equation and the incompressibility. Taking the divergence of Eq. (19) and using the incompressibility condition, we get $\Delta p=0$. Using Eq. (22), this relation yields (here and in the following, the derivative $d / d r$ is denoted by a prime ')

$$
\Delta(\mathbf{G} \cdot \nabla h)=\mathbf{G} \cdot \mathbf{e}_{\mathbf{r}}(\Delta h)^{\prime}=0 .
$$

Thus, introducing the constants $c_{0}, c_{1}$, and $c_{2}$, we have

$$
\Delta h=c_{0} \quad \text { and } \quad h(r)=c_{0} \frac{r^{2}}{6}-c_{1} \frac{1}{r}+c_{2} .
$$

Later on, the constant $c_{2}$ of no physical meaning is set to zero. Using Eq. (22), the incompressibility reads

$$
\begin{aligned}
\nabla \cdot(\mathbf{G} \cdot[\nabla \otimes \nabla f+g \mathbf{I}]) & =\mathbf{G} \cdot \nabla[\Delta f+g] \\
& =\mathbf{G} \cdot \mathbf{e}_{\mathbf{r}}(\Delta f+g)^{\prime}=0 .
\end{aligned}
$$

Consequently, we have

$$
\begin{aligned}
& g=-\Delta f+a_{0} \quad \text { and } \\
& \nabla \otimes \nabla f+g \mathbf{I}=\nabla \otimes \nabla f-\Delta f \mathbf{I}+a_{0} \mathbf{I} .
\end{aligned}
$$

Noting that $\nabla \nabla\left(r^{2}\right)-\Delta\left(r^{2}\right) \mathbf{I}=-4 \mathbf{I}$, the constant $a_{0}$ can be set to zero by including a term $-\left(a_{0} / 4\right) r^{2}$ into $f$. Consequently, $\mathbf{v}$ is expressed from a single potential function $f$,

$$
\mu \mathbf{v}=\mathbf{G} \cdot[\nabla \otimes \nabla f-\mathbf{I} \Delta f] .
$$

Now, inserting expression (24) of $\mathbf{v}$ in the Navier-Stokes equation leads to

$$
\begin{aligned}
\mathbf{0}= & -\nabla \cdot(\mathbf{G} \cdot \nabla h)-\frac{1}{\delta^{2}} \mathbf{G} \cdot[\nabla \otimes \nabla \mathbf{f}-\mathbf{I} \Delta \mathbf{f}] \\
& +\Delta(\mathbf{G} \cdot[\nabla \otimes \nabla f-\mathbf{I} \Delta f]) \\
= & \mathbf{G} \cdot\left[-\mathbf{I} \Delta h+[\nabla \otimes \nabla-\mathbf{I} \Delta]\left[-h+\left(\Delta-\frac{1}{\delta^{2}}\right) f\right]\right] .
\end{aligned}
$$

Therefore, using Eq. (23) and denoting $A=-h+\left(\Delta-1 / \delta^{2}\right) f$, we obtain

$$
\begin{aligned}
& \mathbf{G} \cdot {\left[-c_{0} \mathbf{I}+(\nabla \otimes \nabla-\mathbf{I} \Delta) A\right] } \\
&=\mathbf{G} \cdot\left[-\mathbf{I}\left(c_{0}+\frac{\left(r A^{\prime}\right)^{\prime}}{r}\right)+\mathbf{r} \otimes \mathbf{r} \frac{\left(A^{\prime} / r\right)^{\prime}}{r}\right]=0 .
\end{aligned}
$$

This last equation implies that 


$$
\frac{\left(A^{\prime} / r\right)^{\prime}}{r}=0 \quad \text { and } \quad \frac{\left(r A^{\prime}\right)^{\prime}}{r}=-c_{0}
$$

whose integration gives (since any constant may be incorporated into $h$ without any other change)

$$
A=-h+\left(\Delta-\frac{1}{\delta^{2}}\right) f=-c_{0} \frac{r^{2}}{4} .
$$

Finally, from the expression for $h$ given by Eq. (23), $f$ satisfies the Helmohltz equation (associated with viscous waves) with the following forcing terms:

$$
\left(\Delta-\frac{1}{\delta^{2}}\right) f=-c_{0} \frac{r^{2}}{12}-c_{1} \frac{1}{r} \text {. }
$$

The solution is the sum of particular solutions and spherical wave functions that introduce two additional constants $c$ and $c^{\prime}$,

$$
f=\delta^{2}\left[c_{0}\left(\frac{r^{2}}{12}+\frac{\delta^{2}}{2}\right)+c_{1} \frac{1}{r}+\delta^{2}\left(c \frac{e^{r / \delta}}{r / \delta}+c^{\prime} \frac{e^{-r / \delta}}{-r / \delta}\right)\right] .
$$

To summarize, the general expressions of the pressure $p$ and the velocity $\mathbf{v}$ are given by the set of Eqs. (22)-(24) and (26) that involve four constants. The next step is to define appropriate boundary conditions for the identification of the constants and the dynamic permeability.

\section{Boundary conditions}

The following expressions for the velocity and the shear stress in the direction normal to the surface are useful for writing the boundary conditions:

$$
\begin{aligned}
& \mu \mathbf{v}=\mathbf{G}[\nabla \nabla f-\mathbf{I} \Delta f]=\mathbf{G} \cdot\left[f^{\prime} / r-\Delta f\right]+\mathbf{e}_{\mathbf{r}}(\mathbf{G} \cdot \mathbf{r})\left(f^{\prime} / r\right)^{\prime}, \\
& \mu v_{r}=\left(\mathbf{G} \cdot \mathbf{e}_{\mathbf{r}}\right)\left[f^{\prime} / r-\Delta f+r\left(f^{\prime} / r\right)^{\prime}\right]=-2\left(f^{\prime} / r\right)\left(\mathbf{G} \cdot \mathbf{e}_{\mathbf{r}}\right), \\
& \mu v_{\theta}=\left(\mathbf{G} \cdot \mathbf{e}_{\theta}\right)\left[f^{\prime} / r-\Delta f\right]=-\left[f^{\prime} / r+f^{\prime \prime}\right]\left(\mathbf{G} \cdot \mathbf{e}_{\theta}\right), \\
& 2 \mu \mathbf{D}(\mathbf{v}) \cdot \mathbf{e}_{\mathbf{r}}=-\mathbf{G} \cdot f^{\prime \prime \prime}+\mathbf{e}_{\mathbf{r}}\left(\mathbf{G} \cdot \mathbf{e}_{\mathbf{r}}\right)\left[f^{\prime \prime \prime}+4\left(f^{\prime} / r\right)^{\prime}\right] \\
& 2 \mu D_{r r}=-4\left(f^{\prime} / r\right)^{\prime}\left(\mathbf{G} \cdot \mathbf{e}_{\mathbf{r}}\right), \\
& 2 \mu D_{r \theta}=-f^{\prime \prime \prime}\left(\mathbf{G} \cdot \mathbf{e}_{\theta}\right), \\
& \mu \operatorname{curl}(\mathbf{v})=-[\mathbf{G} \times \mathbf{r}] \frac{(\Delta f)^{\prime}}{r} .
\end{aligned}
$$

a. Conditions for the velocity. At the fluid-solid interface $(r=\beta R)$, the adherence condition $\mathbf{v}(\beta R)=0$ gives, by combining Eqs. (28) and (29),

$$
-2 \frac{f^{\prime}(\beta R)}{\beta R}=0
$$

$$
-\frac{2}{3} \Delta f(\beta R)=0 .
$$

Moreover, the average velocity within the inclusion $\mathcal{I}$ is equal to the velocity in the equivalent Darcy medium. Thus we have

$$
\begin{aligned}
\mathbf{V} & =\frac{1}{\Omega_{\mathcal{I}}} \int_{\Omega_{f}} \mathbf{v} d \Omega=\frac{1}{\Omega} \int_{\partial \Omega_{\mathcal{I}}} \mathbf{r}\left(\mathbf{v} \cdot \mathbf{e}_{r}\right) d s+0 \\
& =-2 \frac{f^{\prime}(R)}{R} \frac{\mathbf{G}}{\mu}=-\frac{K}{\mu} \mathbf{G} .
\end{aligned}
$$

Therefore, we get

$$
-2 \frac{f^{\prime}(R)}{R}+K=0 .
$$

From Eq. (28), this equation also means that the normal velocities in the fluid and in the equivalent Darcy medium are identical at any point on the inclusion boundary, i.e., $v_{r}(R)$ $=V_{r}(R)$.

b. Condition for the stress at the inclusion boundary. The momentum balance in the fluid is not expressed by the Darcy law, and since the viscous stresses are transferred to the skeleton of the porous media it is not possible to impose a priori the pressure nor the stress at the boundary. However, it is physically justified to transpose the result proven by HPM to the present situation: on the boundary of the inclusion the drag force exerted by the corrective stresses $\boldsymbol{\sigma}_{c}$-i.e., the difference between the stress in the fluid inclusion and the pressure in the equivalent Darcy mediumequals zero. This means that the inclusion equilibrium is satisfied on average by the stress of zero order. This leads to

$$
\int_{\partial \Omega_{\mathcal{I}}} \boldsymbol{\sigma}_{c} \mathbf{n} d s=0 \quad \text { with } \quad \boldsymbol{\sigma}_{c}=-(p-P) \mathbf{I}+2 \mu \mathbf{D}(\mathbf{v}) .
$$

Introducing the pressure expressions and the shear stress components, then integrating, gives

$$
\begin{aligned}
0= & \int_{\partial \Omega_{\mathcal{I}}}(\mathbf{G} \cdot \mathbf{r})\left(-h^{\prime}+1\right) \mathbf{e}_{\mathbf{r}} \\
& +\left(-\mathbf{G} \cdot f^{\prime \prime \prime}+\mathbf{e}_{\mathbf{r}}\left(\mathbf{G} \cdot \mathbf{e}_{\mathbf{r}}\right)\left[f^{\prime \prime \prime}-4\left(f^{\prime} / r\right)^{\prime}\right]\right) \cdot \mathbf{e}_{\mathbf{r}} d s \\
= & \frac{4 \pi R^{2}}{3} \mathbf{G}\left[-h^{\prime}+R+f^{\prime \prime \prime}-4\left(f^{\prime} / r\right)^{\prime}-3 f^{\prime \prime \prime}\right] \\
= & \frac{4 \pi R^{2}}{3} \mathbf{G}\left[-h^{\prime}+R-2(\Delta f)^{\prime}\right] .
\end{aligned}
$$

Thus the equation expressing the overall equilibrium reads

$$
-h^{\prime}(R)+R-2(\Delta f)^{\prime}(R)=0 .
$$

c. Micro-macro energy consistency. It remains to express the energy equivalence between the inclusion and the same volume of the equivalent Darcy medium. According to Eq. (16) derived by HPM, the conservation of energy throughout upscaling is written as 


$$
\int_{\Omega_{f}}\left[2 \mu \mathbf{D}_{y}(\mathbf{v}): \mathbf{D}_{y}(\overline{\mathbf{v}})+i \omega \rho \mathbf{v} \cdot \overline{\mathbf{v}}\right] d \Omega=-\nabla P \cdot \int_{\Omega_{f}} \overline{\mathbf{v}} d \Omega .
$$

Now, following the same reasoning as in Sec. II B 3, taking the scalar product of the momentum balance equation [Eq. (19)] with the field $\overline{\mathbf{v}}$, and integrating over the pores' volume, we obtain

$$
\begin{aligned}
\int_{\Omega_{f}} & {[2 \mu \mathbf{D}(\mathbf{v}): \mathbf{D}(\overline{\mathbf{v}})+i \omega \rho \mathbf{v} \cdot \overline{\mathbf{v}}] d \Omega } \\
= & \int_{\partial \Omega_{\mathcal{I}}}\left[-p \mathbf{e}_{\mathbf{r}}+2 \mu \mathbf{D}(\mathbf{v}) \mathbf{e}_{\mathbf{r}}\right] \cdot \overline{\mathbf{v}} d s .
\end{aligned}
$$

By comparison with the previous equality, the following relation must be fulfilled:

$$
\int_{\partial \Omega_{\mathcal{I}}}\left[-p \mathbf{e}_{\mathbf{r}}+2 \mu \mathbf{D}(\mathbf{v}) \mathbf{e}_{\mathbf{r}}\right] \cdot \overline{\mathbf{v}} d s=-\nabla P \cdot \int_{\Omega_{f}} \overline{\mathbf{v}} d \Omega .
$$

Note that the right hand side term may be changed into

$$
\begin{aligned}
-\nabla P \cdot \int_{\Omega_{f}} \overline{\mathbf{v}} d \Omega & =-\nabla P \cdot \int_{\partial \Omega_{\mathcal{I}}} \mathbf{r}\left(\overline{\mathbf{v}} \cdot \mathbf{e}_{\mathbf{r}}\right) d s \\
& =-\int_{\partial \Omega_{\mathcal{I}}} P\left(\overline{\mathbf{v}} \cdot \mathbf{e}_{\mathbf{r}}\right) d s,
\end{aligned}
$$

so that combined with the overall equilibrium condition [Eq. (37)] and multiplied by the conjugate of the mean velocity $\overline{\mathbf{V}}$, the energy equivalence takes the form

$$
\int_{\partial \Omega_{\mathcal{I}}}\left[(P-p) \mathbf{e}_{\mathbf{r}}+2 \mu \mathbf{D}(\mathbf{v}) \cdot \mathbf{e}_{\mathbf{r}}\right] \cdot[\overline{\mathbf{v}}-\overline{\mathbf{V}}] d s=0 .
$$

And since the normal velocities are identical on $\partial \Omega_{\mathcal{I}}$, see Eq. (36), one obtains

$$
\int_{\partial \Omega_{\mathcal{I}}} 2 \mu D_{r \theta}\left[\bar{v}_{\theta}-\bar{V}_{\theta}\right] d s=0 .
$$

According to the field expressions, one gets two possibilities on the boundary $(r=R)$ :

- The shear stress $D_{r \theta}$ vanishes uniformly, and therefore,

$$
f^{\prime \prime \prime}(R)=0 \text {. }
$$

In that case, on the inclusion boundary, the stress in the normal direction in the fluid equals the pressure in the equivalent Darcy medium, i.e., $\sigma(R) \cdot \mathbf{e}_{\mathrm{r}}=P(R) \mathbf{e}_{\mathrm{r}}$.

- The tangential velocities are equal, i.e., $v_{\theta}=V_{\theta}$, giving,

$$
-\frac{2}{3} \Delta f(R)+K=0 .
$$

In that case the velocities in the fluid and in the equivalent Darcy medium are identical on the inclusion boundary, i.e., $\mathbf{v}(R)=\mathbf{V}(R)$. It can also be derived that $D_{r r}(R)=0$.

To summarize, two alternative sets of five conditions are obtained [Eqs. (34)-(36), (38), and (40) or Eqs. (34)-(36), (38), and (41)]. Four of them are common to both sets, i.e., the two conditions related to the adherence condition, the condition expressing the identity between the normal microvelocity and the Darcy flow, and the averaged or overall equilibrium condition. The remaining equation is derived from the energy equivalence. Thus two possibilities are consistent:

- The shear stress vanishes, and the stress vector at the boundary of the fluid inclusion matches the pressure in the Darcy medium, which corresponds to the assumption made by Berdichevsky and Cai. ${ }^{9}$ This will be called the pressure approach (denoted as P-) in the following. The tangential microvelocity is not continuous with the tangential Darcy flow.

- The microvelocity matches the Darcy flow, and neither the pressure nor the stress vector at the boundary of the fluid inclusion matches the pressure in the equivalent media. This will be called the flow approach (denoted as V-).

d. Remark about the "zero vorticity assumption." The assumption used by Tarnow ${ }^{11}$ and Umnova et al. ${ }^{15}$ of vanishing vorticity at the boundary of the cell (denoted hereafter as C-estimate) does not arise from the SCM and thus transgresses the energy consistency. Despite this drawback, considerations on the kinematics of the flow at the boundary of identical cells argue in favor of the zero vorticity assumption. Indeed, the level of vorticity is the highest in the narrow gaps between particles but vanishes when the velocity reaches its maximum at the middle of the gap, i.e., at the boundary of the cell. According to Eq. (33), this assumption leads to

$$
(\Delta f)^{\prime}(R)=0 .
$$

Moreover, combined with overall equilibrium [Eq. (38)], one obtains

$$
-h^{\prime}(R)+R=0,
$$

meaning that (i) the pressure (not the stress) at the boundary of the fluid equals the pressure in the Darcy medium and (ii) the dissipated energy due to $\mathbf{v}-\mathbf{V}$ on the surface is neglected. The P- and C-assumptions may be expected to give more reliable results because, referring to the HPM approach, the $\mathrm{P}$ - and C-boundary conditions are correct up to the first order, which is not the case for the V-boundary conditions. In the following, the three approaches are investigated.

\section{B. Resolution and estimates}

Expressions (23)-(26) for functions $h$ and $f$ are introduced in the two possible sets of five conditions. This leads to two linear systems whose resolution allows the determination in both cases of the five parameters $\left(c_{0}, c_{1}, c, c^{\prime}\right.$, and $K)$.

\section{Pressure approach: Fields $\mathrm{v}_{p}=\varpi_{p}$ and $p_{p}$}

Writing the complex number $x=R / \delta$, with $\delta=\sqrt{\mu / i \rho \omega}$, the linear system [Eqs. (34), (35), (40), (36), and (38)] reads

$$
c \frac{e^{\beta x}}{\beta x}+c^{\prime} \frac{e^{-\beta x}}{-\beta x}+0+\frac{c_{0}}{2}+0=0,
$$




$$
\begin{aligned}
& c \frac{1}{\beta x}\left(1-\frac{1}{\beta x}\right) \frac{e^{\beta x}}{\beta x}-c^{\prime} \frac{1}{\beta x}\left(1+\frac{1}{\beta x}\right) \frac{e^{-\beta x}}{-\beta x}-\frac{c_{1}}{(\beta R)^{3}}+\frac{c_{0}}{6} \\
& +0=0, \\
& c\left[\left(1+\frac{6}{x^{2}}\right)(x-1)-2\right] \frac{e^{x}}{x}-c^{\prime}\left[\left(1+\frac{6}{x^{2}}\right)(x+1)\right. \\
& +2] \frac{e^{-x}}{-x}-\frac{6 c_{1}}{R^{3}}+0+0=0, \\
& -c \frac{1}{x}\left(1-\frac{1}{x}\right) \frac{e^{x}}{x}+c^{\prime} \frac{1}{x}\left(1+\frac{1}{x}\right) \frac{e^{-x}}{-x}-\frac{c_{1}}{2 R^{3}}-\frac{c_{0}}{6}+0=\frac{1}{2}, \\
& \frac{3 c_{1}}{R^{3}}+0-\frac{K_{p}}{\delta^{2}}=0 .
\end{aligned}
$$

The analytical solution leads to the following permeability P-estimate, denoted as $K_{p}$ :

$$
K_{p}=\frac{\delta^{2}}{1-3 / x^{2} C_{p}}, \quad C_{p}=\frac{A_{p}+B_{p} \tanh [x(\beta-1)] / x}{a_{p}+b_{p} \tanh [x(\beta-1)] / x},
$$

where

$$
\begin{aligned}
A_{p}= & {\left[3+(\beta x)^{2}\right]\left[1+x^{2} / 6\right]-3 \beta\left[1+x^{2} / 2\right], } \\
B_{p}= & {\left[3+(\beta x)^{2}\right]\left[1+x^{2} / 2\right]-3 \beta x^{2}\left[1+x^{2} / 6\right], } \\
a_{p}= & {\left[3+(\beta x)^{2}\right] / 3-3 \beta-\left[1+x^{2} / 6\right] 2 / \beta } \\
& +\frac{4}{\cosh [x(\beta-1)]}, \\
b_{p}= & {\left[3+(\beta x)^{2}\right]-\beta x^{2}-\left[1+x^{2} / 2\right] 2 / \beta . }
\end{aligned}
$$

The reciprocal of $K_{p}, H_{p}$, takes the form

$$
H_{p}=\frac{1}{\delta^{2}}\left[1-\frac{3}{x^{2}} C_{p}\right] \text {. }
$$

In addition, from the coefficients $c, c^{\prime}, c_{0}$, and $c_{1}$ (not detailed here), which are solutions of the system of equations [Eqs. (34), (35), (40), (36), and (38)], one builds the velocity field $\mathbf{v}_{p}=\boldsymbol{\varpi}_{p}$. This field is the exact solution for the flow through the fluid shell under the imposed pressure conditions at the external boundary $(r=R): \boldsymbol{\sigma} \mathbf{n}=-P \mathbf{n}$, with $\nabla P=\mathbf{e}_{\mathbf{z}}$.

\section{Flow approach: Fields $\mathrm{v}_{v}=\varpi_{v}$ and $p_{v}$}

For this approach, the set of equations [Eqs. (34), (35), (41), (36), and (38)] to be solved is

$$
\begin{aligned}
& c \frac{e^{\beta x}}{\beta x}+c^{\prime} \frac{e^{-\beta x}}{-\beta x}+0+\frac{c_{0}}{2}+0=0, \\
& c \frac{1}{\beta x}\left(1-\frac{1}{\beta x}\right) \frac{e^{\beta x}}{\beta x}-c^{\prime} \frac{1}{\beta x}\left(1+\frac{1}{\beta x}\right) \frac{e^{-\beta x}}{-\beta x}-\frac{c_{1}}{(\beta R)^{3}}+\frac{c_{0}}{6} \\
& +0=0, \\
& c \frac{e^{x}}{x}+c^{\prime} \frac{e^{-x}}{-x}+0+\frac{c_{0}}{2}+\frac{3 K_{v}}{2 \delta^{2}}=0,
\end{aligned}
$$

$$
\begin{aligned}
& -c \frac{1}{x}\left(1-\frac{1}{x}\right) \frac{e^{x}}{x}+c^{\prime} \frac{1}{x}\left(1+\frac{1}{x}\right) \frac{e^{-x}}{-x}-\frac{c_{1}}{2 R^{3}}-\frac{c_{0}}{6}+0=\frac{1}{2}, \\
& \frac{3 c_{1}}{R^{3}}+0-\frac{K_{v}}{\delta^{2}}=0 .
\end{aligned}
$$

The analytical solution reads

$$
K_{v}=\frac{\delta^{2}}{1-3 / x^{2} C_{v}}, \quad C_{v}=\frac{A_{v}+B_{v} \tanh [x(\beta-1)] / x}{a_{v}+b_{v} \tanh [x(\beta-1)] / x},
$$

where

$$
\begin{aligned}
& A_{v}=\left[3+(\beta x)^{2}\right]-3 \beta\left[1+x^{2} / 3\right], \\
& B_{v}=\left[3+(\beta x)^{2}\right]\left[1+x^{2} / 3\right]-3 \beta x^{2}, \\
& a_{v}=-2[\beta+1 / \beta]+\frac{4}{\cosh [x(\beta-1)]}, \\
& b_{v}=\left[3+(\beta x)^{2}\right] 2 / 3-\left[1+x^{2} / 3\right] 2 / \beta .
\end{aligned}
$$

The reciprocal of $K_{v}, H_{v}$, takes the form

$$
H_{v}=\frac{1}{\delta^{2}}\left[1-\frac{3}{x^{2}} C_{v}\right] \text {. }
$$

The coefficients $c, c^{\prime}, c_{0}$, and $c_{1}$ (not detailed here), which are solutions of the system of Eqs. (34), (35), (41), (36), and (38), defines the velocity field $\mathbf{v}_{v}=\boldsymbol{\varpi}_{v}$. The field $\boldsymbol{\varpi}_{v} \mu H_{v}$ is the exact solution for the flow through the fluid shell under the imposed unit flow condition at the external boundary $(r=R): \boldsymbol{\varpi}_{v} \mu H_{v}=-\mathbf{e}_{\mathbf{z}}$.

It is worth noting that embedded in a medium of permeability $K_{p}$ (or $K_{v}$ ), the inclusion $\mathcal{I}\{\beta, R\}$ is almost neutral in the sense that its presence modifies neither the average density of dissipated and kinetic powers nor the field in the Darcy medium and that the condition of stress (or the flow) continuity is satisfied.

\section{Zero vorticity approach: C-estimate}

Similarly, the resolution of the set of Eqs. (34), (35), (42), (36), and (38) leads to the following $K_{c}$-estimate:

$$
\begin{aligned}
K_{c}= & 2 \delta^{2}\left(1-\frac{1}{1-A_{c}}\right), \quad A_{c}=\frac{\left(1-\beta^{3}\right)}{3} \\
& +\frac{\beta^{2}}{x^{2}} \frac{1-1 / \beta+\left(x^{2}-1 / \beta\right) \tanh ((\beta-1) x) / x}{1+\tanh ((\beta-1) x) / x} .
\end{aligned}
$$

This expression differs from that given by Umnova et al. ${ }^{15}$ [in this latter case, it seems that the porosity $\phi$ is missing in the right hand of expression (12) of $D]$.

\section{Comments}

a. Low frequency. Expanding the dynamic permeability at low frequency gives a behavior in conformity to Eq. (12) whose coefficients correspond to the intrinsic permeability and the added mass effect $\alpha_{0}$ of the Darcy medium P- or $\mathrm{V}$-consistent with the inclusion $\mathcal{I}\{\beta, R\}$. The intrinsic permeabilities are 

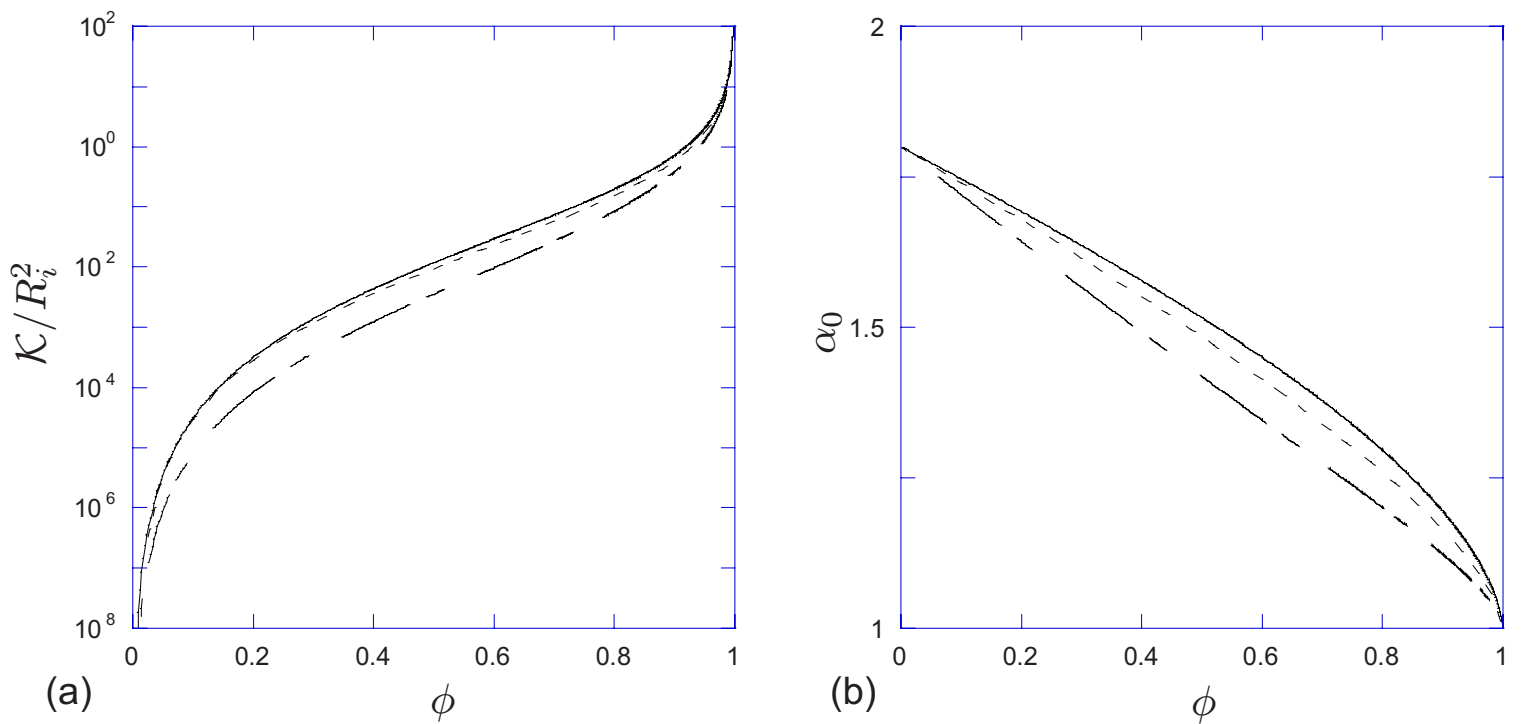

FIG. 3. (Color online) Low frequency parameters. (a) Dependence of dimensionless estimates $\mathcal{K}_{p} / R_{i}^{2}$ (P-estimate, continuous line), $\mathcal{K}_{v} / R_{i}^{2}(\mathrm{~V}$-estimate, mixed line), and $\mathcal{K}_{c} / R_{i}^{2}$ (C-estimate, dashed line) on the porosity. (b) Dependence of the low frequency tortuosities $\alpha_{0 p}, \alpha_{0 v}$, and $\alpha_{0 c}$ on the porosity.

$$
\begin{aligned}
& \mathcal{K}_{p}=\frac{1}{3}\left[-1+\frac{2+3 \beta^{5}}{\beta\left(3+\beta^{5}\right)}\right] R^{2}, \\
& \mathcal{K}_{v}=\frac{1}{18}\left[4 \frac{1-\beta}{\beta}-5 \frac{\left(1-\beta^{2}\right)^{2}}{1-\beta^{5}}\right] R^{2},
\end{aligned}
$$

where $R=\beta^{-1} R_{i}$. These values coincide with those established for permanent flow in Boutin. ${ }^{10}$ Concerning the $\mathrm{C}$-estimate, the static permeability is written as

$$
\mathcal{K}_{c}=\frac{2}{45}\left[\frac{5-9 \beta+5 \beta^{3}-\beta^{6}}{\beta}\right] R^{2} .
$$

Figure 3(a) shows the dependence of the three dimensionless estimates on the porosity $\phi=1-\beta^{3}$. This figure underlines the tremendous effect of the porosity on the intrinsic permeability, while the ratio $\mathcal{K}_{p} / \mathcal{K}_{v}$ varies from 1 (dilute solid concentration, i.e., large porosity) to 4 (dense solid concentration, i.e., small porosity). We note that the ratio $\mathcal{K}_{p} / \mathcal{K}_{c}$ reaches a maximum of about 1.3 when $\phi \approx 3 / 4$.

The analytical expressions for the low frequency tortuosity, which depends on $\beta$ only, are rather complicated and not presented here. Figure 3(b) shows the dependence of the three tortuosities, $\alpha_{0_{p}}, \alpha_{0_{v}}$, and $\alpha_{0_{c}}$, on the porosity $\phi$. The values lie in a narrow range $[1 ; 9 / 5]$; moreover $\alpha_{0_{p}} / \alpha_{0_{v}}>1$ and $\alpha_{0_{p}} / \alpha_{0_{c}}>1$.

$b$. High frequency. The high frequency behavior derived by expanding the dynamic permeabilities complies with Eq. (13). It provides the added mass effect $\alpha_{\infty}$ (or tortuosity) and the form factor $M$. For the tortuosity, the $\mathrm{P}-, \mathrm{V}-$ and C-estimates lead to the same value,

$$
\alpha_{\infty p, v, c}=1+\beta^{3} / 2=\frac{3-\phi}{2}<\alpha_{0 v}<\alpha_{0 c}<\alpha_{0 p} .
$$

The reason for this coincidence lies in the fact that at high frequencies, the viscous effects are much smaller than the inertia. Thus, the tortuosity $\alpha_{\infty}$ may be derived from a perfect fluid flow, where the interface conditions involve only the radial component $\mathbf{v} \cdot \mathbf{n}$, so that the three approaches give the same results.

This latter is identical to a thermal problem ${ }^{19}$ where the inverse of $i \omega \rho$ plays the role of thermal conductivity (the solid can be considered of infinite density, i.e., of zero thermal conductivity). Transposing Hashin's results in terms of tortuosity leads to the above result. Figure 4(a) shows the dependence of the high frequency tortuosity $\alpha_{\infty \propto p, v, c}$ on the porosity.

Using the low and high frequency limits, the P-, V-, and $\mathrm{C}$-equivalent media present different critical frequencies.

$$
\omega_{c i}=\frac{\mu \phi}{\mathcal{K}_{i} \rho \alpha_{\infty}}, \quad i=p, v, c .
$$

Finally, the form factors are written as

$$
\begin{aligned}
& M_{p}=2\left[\frac{9 \beta^{2}}{2 \phi^{2}}\right]^{2} \frac{\phi \mathcal{K}_{p}}{\alpha_{\infty} R^{2}}, \quad M_{v}=2\left[\frac{9\left(\beta^{2}+\beta^{6}\right)}{2 \phi^{2}}\right]^{2} \frac{\phi \mathcal{K}_{v}}{\alpha_{\infty} R^{2}}, \\
& M_{c}=2\left[\frac{9 \beta^{3}}{2 \phi^{2}}\right]^{2} \frac{\phi \mathcal{K}_{c}}{\alpha_{\infty} R^{2}} .
\end{aligned}
$$

In terms of characteristic viscous length, one obtains

$$
\frac{\Lambda_{p}}{R_{i}}=\frac{\Lambda_{c}}{R_{i}}=\frac{4 \phi}{9(1-\phi)} \alpha_{\infty}=\frac{2 \phi(3-\phi)}{9(1-\phi)}, \quad \Lambda_{v}=\frac{\Lambda_{p}}{1+\beta^{4}},
$$

Figure 4(b) shows the dependence of form factors $M_{p}, M_{v}$, and $M_{c}$ on the porosity. These coefficients vary from 0 at high porosity to $2 / 3$ at low porosity, and $1<M_{p} / M_{v}<2$.

c. Whole frequency range. The dependencies of $K_{p}, K_{v}$, and $K_{c}$ and $H_{p}, H_{v}$, and $H_{c}$ on the dimensionless frequency $\left(\omega / \omega_{c p}\right)$ are presented in Figs. 5 and 6, respectively, for a porosity $\phi=1 / 3$. Figure 5 shows that three estimates comply with properties (12) and (13) of the dynamic permeability. Figure 6 shows the dependence of the real and imaginary parts of the dimensionless estimates on $\omega / \omega_{c}$. Figure 6 highlights the following inequalities: 

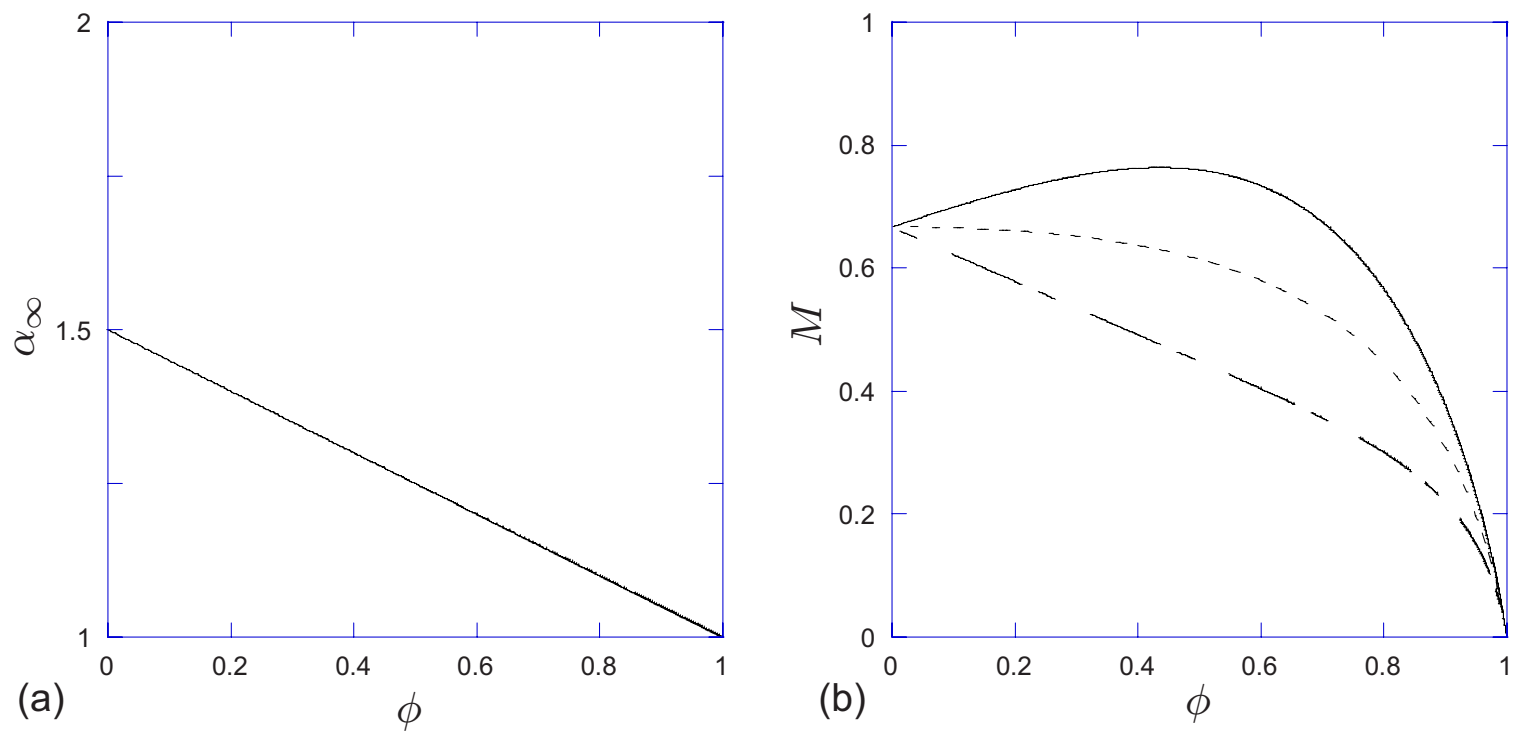

FIG. 4. (Color online) High frequency parameters. (a) Dependence of high frequency tortuosities $\alpha_{\infty \sim p, v, c}$ on the porosity. (b) Dependence of form factors $M_{p}$ (continuous line), $M_{v}$ (mixed line), and $M_{c}$ (dashed line) on the porosity.

$$
\begin{aligned}
& \mathcal{K}_{p} H_{p}^{R}\left(\omega / \omega_{c p}\right) \geqslant \mathcal{K}_{v} H_{v}^{R}\left(\omega / \omega_{c v}\right), \\
& \frac{\phi \mu}{\omega \rho} H_{p}^{I}\left(\omega / \omega_{c p}\right) \geqslant \frac{\phi \mu}{\omega \rho} H_{v}^{I}\left(\omega / \omega_{c v}\right) .
\end{aligned}
$$

The dimensionless curves present the same main qualitative features and, according to the theoretical result [Eq. (17)], $H^{R}$ increases monotonically versus frequency and $H^{I} / \omega$ decreases.

Differences (related to the P-, V-, and C-approaches and to the porosity) are noticeable, quantitatively, for the viscous effect at high frequencies and the added mass at low frequencies. The difference in the critical frequencies of both approaches makes the discrepancy magnified for the dimensional coefficients.

To conclude, the three estimates present the same qualitative features. The $\mathrm{C}$-estimate lie between the P-estimate and the $\mathrm{V}$-estimate in the whole porosity range and predict a similar behavior as the P-estimate.

d. Comparison with the models of Johnson et al. and Pride et al.. The formula giving the frequency dependencies of the dynamic permeability, proposed by Johnson et al. ${ }^{3}$ and Pride et al., ${ }^{22}$ require to be applied the knowledge of $\mathcal{K}, M$, and $\alpha_{\infty}$ (and $\alpha_{0}$ with the correction of Pride et al.). This limitation is overcome in our approach since the microstructure knowledge is sufficient to assess analytically the dynamic permeability in the whole frequency range. Thus $\mathcal{K}$, $M, \alpha_{\infty}$, and $\alpha_{0}$ are also analytically derived. As mentioned above, $K_{p}, K_{v}$, and $K_{c}$ follow the general features of any parameter $K(\omega)$ at low and high frequencies. The formula of Johnson et al. is elaborated to match three aspects of the two asymptotic behaviors (i.e., low and high frequency dissipation and high frequency inertia). Thus, provided that the values of $\left(\mathcal{K}, M, \alpha_{\infty}\right)$ obtained in the present paper are used, the formula of Johnson et al. would naturally give correct approximations of $K_{p}, K_{v}$, and $K_{c}$ if one only focuses on three aspects of the low and high frequency asymptotic behaviors. This will be also the case for the four aspects of the asymptotic behavior when using the formula of Pride et al. Note also that the expressions of $K_{p}, K_{v}$, and $K_{c}$ are issued the solution of physical problems, and therefore they are causal functions by construction. If this is also the case for the formula of Johnson et al., it remains an open question for the formula of Pride et al.

\section{FROM SOLID-FLUID INCLUSION TO POROUS MEDIA}

The determination of the equivalent parameter usually constitutes the ultimate step of a self-consistent approach. The obtained value is assumed to be the effective coefficient of heterogeneous media characterized by inclusions $\mathcal{I}$. The present case requires a more detailed attention since

- different values that seem physically acceptable have been obtained,

- conversely to other physical problems (elasticity, conductivity, etc.), the real part of $K$ depends on the size of the inclusion (in addition to the usual dependence on the concentration of the constituents), and

- the local physics that mixes dissipative process and kinetic energy involves an internal intrinsic length (the viscous layer thickness).

Below is discussed how these results apply by examining first the reliability of the estimates for the periodic array of solid spheres and, second, by establishing bounds for polydisperse sphere packing.

\section{A. Dynamic permeability of periodic arrays of solid overlapping and nonoverlapping spheres}

A usual exploitation of the SCM estimates is to consider that they provide a reasonable assessment of the macroscopic properties of sphere packing presenting the same fluid-solid concentration as the generic inclusion. This assumption results from a geometrical approximation that leads to compare 

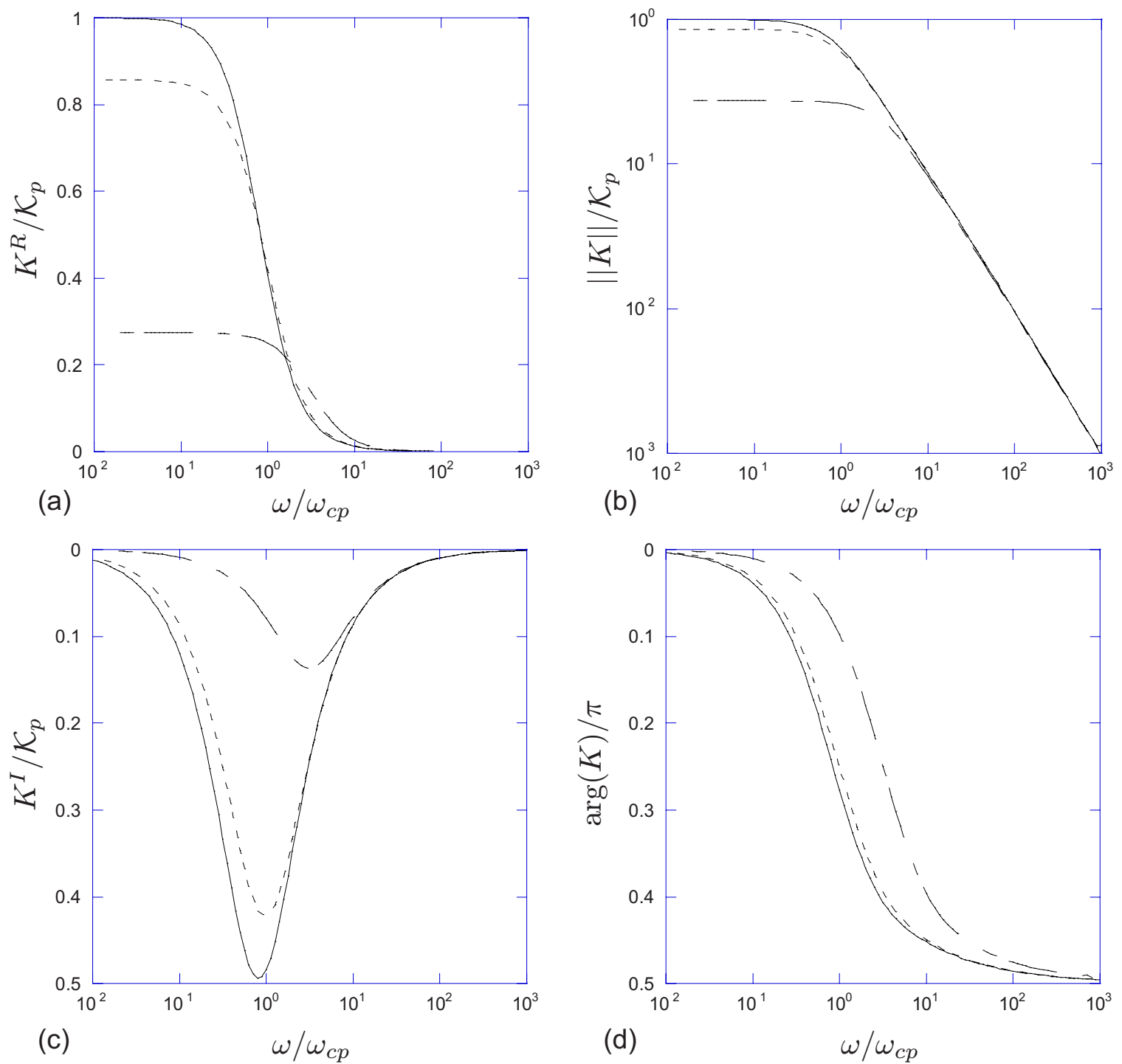

(b)

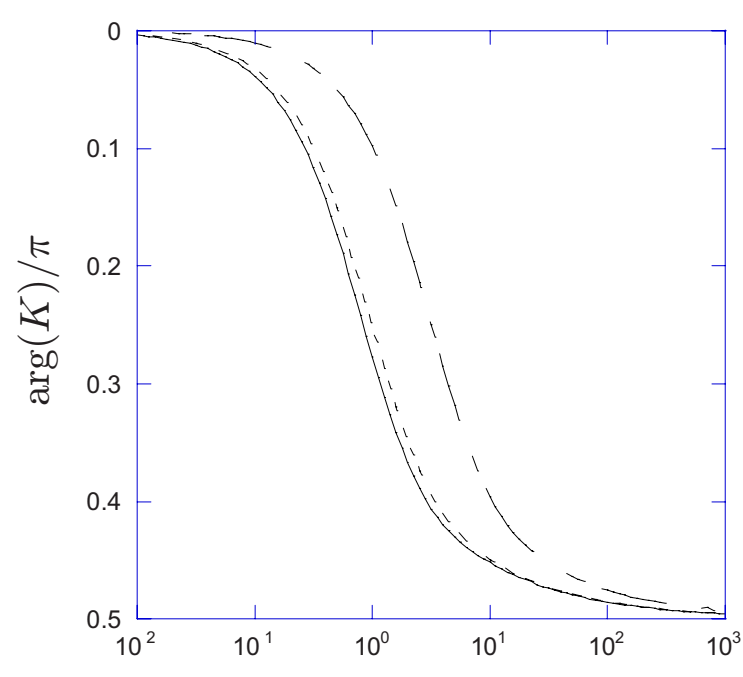

(d)

$$
\omega / \omega_{c p}
$$

FIG. 5. (Color online) Dynamic permeability estimates $K_{p}, K_{v}$, and $K_{c}$ vs $\omega / \omega_{c p}$ when the porosity $\phi=1 / 3$. (a) Real part normalized by the intrinsic permeability $\mathcal{K}_{p}$, (b) modulus normalized by $\mathcal{K}_{p}$, (c) imaginary part normalized by $\mathcal{K}_{p}$, and (d) phase normalized by $\pi$. Note that the difference in intrinsic permeability induces a shift between the P-, V-, and C-curves corresponding to the shift in critical frequencies.

the periodic cell to the spherical inclusion. The objective of this section is to check how far the analytical estimates agree with finite element numerical results obtained by solving boundary value problems arising from the homogenization process on the representative elementary volume (REV) of simple cubic (sc), body-centered cubic (bcc), and facecentered cubic (fcc) arrays of nonoverlapping and overlapping spheres with the same radius $R_{i}$. These periodic lattices of spheres are ranging from dilute systems with isolated spheres and highly concentrated consolidated media. Let us remark that the transition between nonoverlapping and overlapping spheres occurs when the porosity values are equal to $0.47,0.32$, and 0.26 for the sc, bcc, and fcc microstructures, respectively. Due to the symmetry properties of each microstructure, finite simulations have been performed on 1/16 of the total REV. Figure 7 shows typical meshes used to perform finite element simulations. ${ }^{23}$ Figure 8 underlines the influence of the ratio $\omega / \omega_{c}$ on the microscopic velocity field deduced from numerical simulations in a sc array of nonoverlapping spheres $(\phi=0.3)$. We can observe that by in- creasing $\omega / \omega_{c}$, inertial effects dominate and viscous effects are confined to a thin viscous layer around the spheres.

a. Low frequency. At low frequencies, the static permeability and the low frequency tortuosity are obtained by solving the boundary value $S_{v}^{0}$ (Sec. II B 2) when $\omega=0$ for a given macroscopic pressure gradient $\nabla_{x} P$. The microscopic velocity field $\mathbf{v}^{0}$, solution of $S_{v}^{0}$, is in the form

$$
\mathbf{v}^{0}(\mathbf{x}, \mathbf{y})=-\frac{1}{\mu} \mathbf{k} \cdot \nabla_{x} P
$$

where $\mathbf{k}$ is a second order tensor. The static permeability tensor is defined as

$$
\mathbf{K}(\omega=0)=\frac{1}{\Omega} \int_{\Omega_{f}} \mathbf{k} d \Omega=\phi\langle\mathbf{k}\rangle .
$$

From relations (12), (16), and (50), it can be shown that the low frequency tortuosity tensor $\boldsymbol{\alpha}_{0}$ is written as 

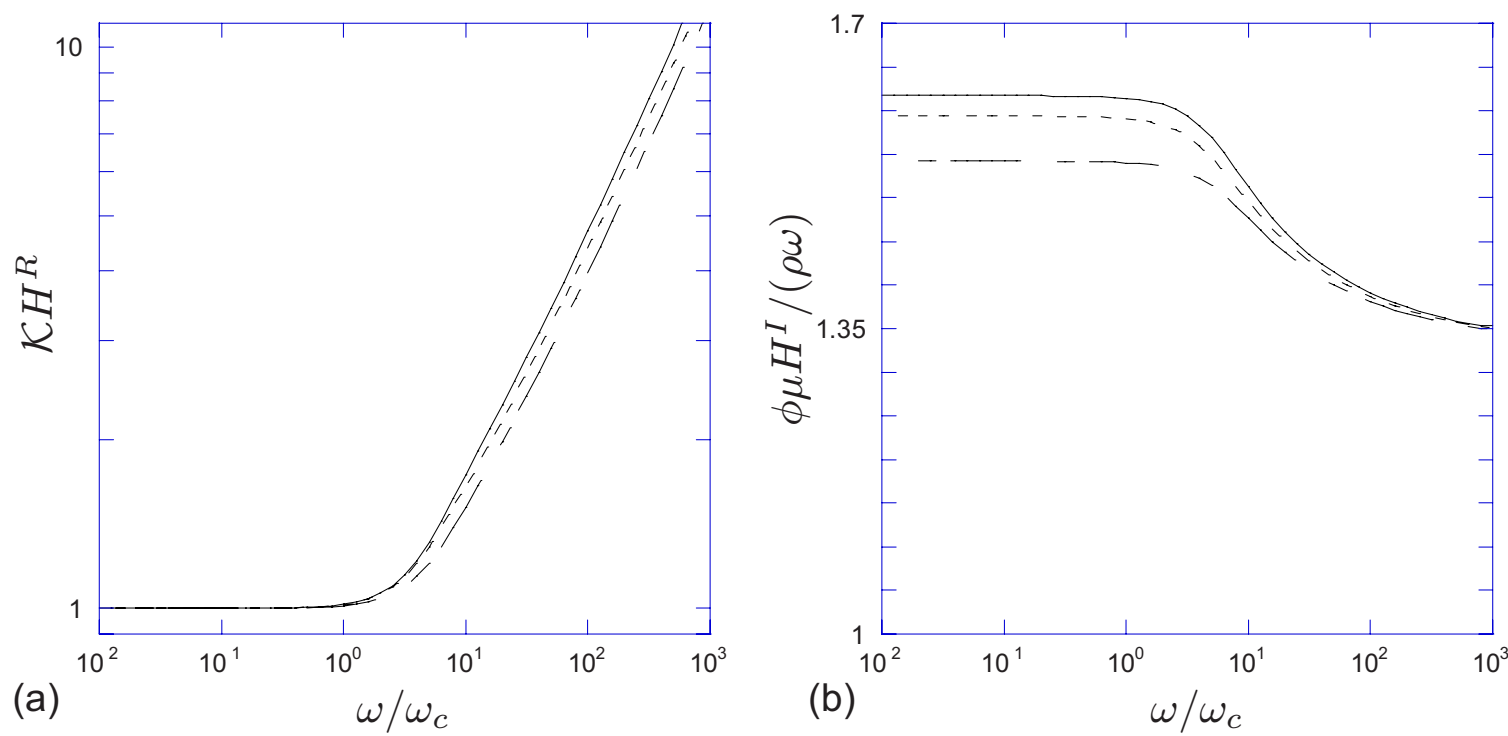

FIG. 6. (Color online) Dynamic estimates $H_{p}$ (continuous line), $H_{v}$ (mixed line), and $H_{c}$ (dashed line) vs $\omega / \omega_{c}$ for a porosity $\phi=1 / 3$ : (a) Dependence of $\mathcal{K}_{p} H_{p}^{R} / \mu, \mathcal{K}_{v} H_{v}^{R} / \mu$, and $\mathcal{K}_{c} H_{c}^{R} / \mu$ on the dimensionless frequency $\omega / \omega_{c}$, where $\omega_{c}$ equals $\omega_{c p}, \omega_{c v}$, and $\omega_{c c}$, respectively. (b) Dependence of $\phi \mu H_{p}^{I} /(\rho \omega)$, $\phi \mu H_{v}^{I} /(\rho \omega)$, and $\phi \mu H_{c}^{I}(\rho \omega)$ on $\omega / \omega_{c}$.

$$
\boldsymbol{\alpha}_{0}=\langle\mathbf{k} \cdot \mathbf{k}\rangle(\langle\mathbf{k}\rangle \cdot\langle\mathbf{k}\rangle)^{-1} .
$$

The symmetry properties of the microstructures under consideration imply that the tensors in such porous media are isotropic. Thus $\mathbf{K}(\omega=0)=\mathcal{K} \mathbf{I}$ and $\boldsymbol{\alpha}_{0}=\alpha_{0} \mathbf{I}$. Figures 9(a) and 9(b) present the dependence with the porosity of the static dimensionless permeability $\mathcal{K} / R_{i}^{2}$ and the low frequency tortuosity $\alpha_{0}$ for sc, bcc, and fcc microstructures.

On both figures, self-consistent estimates (P-estimate, V-estimate, and C-estimate) are also reported. Figure 9(a) shows that the computed dimensionless static permeabilities mainly depend on the porosity. They slightly depend on the arrangement of spheres when the porosity is smaller than 0.4. As expected, the permeability of the periodic arrays is closer to $\mathrm{P}$ - and $\mathrm{C}$-estimates than to the $\mathrm{V}$-estimate. $\mathrm{P}$ - and $\mathrm{C}$-estimates appear as accurate analytical approximations up to the maximum packing concentration. Because of the weak dependence on the sphere arrangement (conversely to the case of fibrous media, Berdichevsky and $\mathrm{Cai}^{9}$ ), these estimates should also be relevant for media with uniform grain
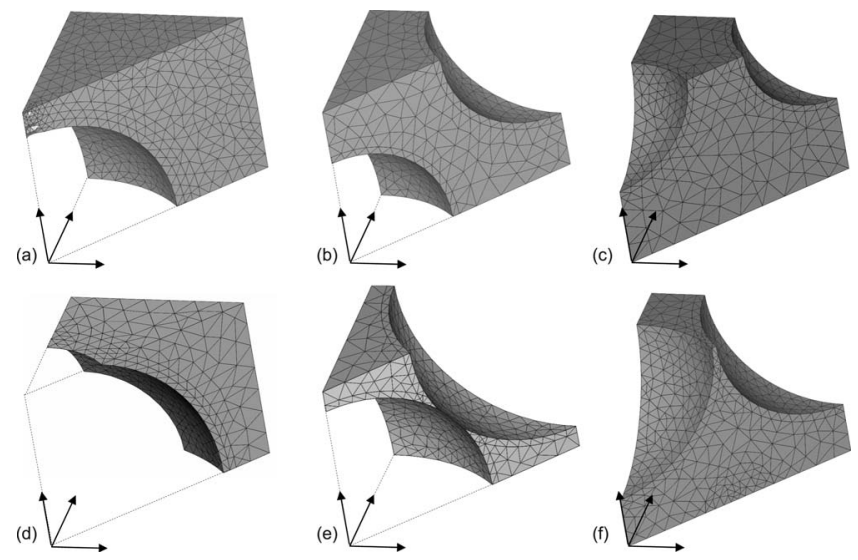

FIG. 7. Typical meshes of [(a) and (d)] sc, [(b) and (e)] bcc, and [(c) and (f)] fcc arrays $(1 / 16$ of the total REV) used to perform finite element simulations. (a)-(c) $\phi=0.7$. (b)-(d) $\phi=0.3$. distribution. Beyond, as explained by Chapman and Higdon, ${ }^{14}$ the geometry of the flow path changes significantly, so that the discrepancies increase. Figure 9(b) shows that the low frequency tortuosity $\alpha_{0}$ for the sc, bcc, and fcc microstructures mainly track the $\mathrm{P}$ - and $\mathrm{C}$-estimates when the porosity is larger than 0.6 . When the porosity is lower than 0.6, P-, V-, and C-approximations underestimate numerical values.

b. High frequency. Figure 10 depicts the dependence of the high frequency tortuosity $\alpha_{\infty}$ and the form factor $M$ on the porosity. In this figure, numerical results for the sc, bcc, and fcc microstructures are compared with the different estimates. Once again, a good agreement of P- and C-estimates with numerical results is observed when the porosity is larger than 0.6. When the porosity is lower than $0.6, \mathrm{P}-, \mathrm{V}-$, and $\mathrm{C}$-approximations underestimate numerical values, specially
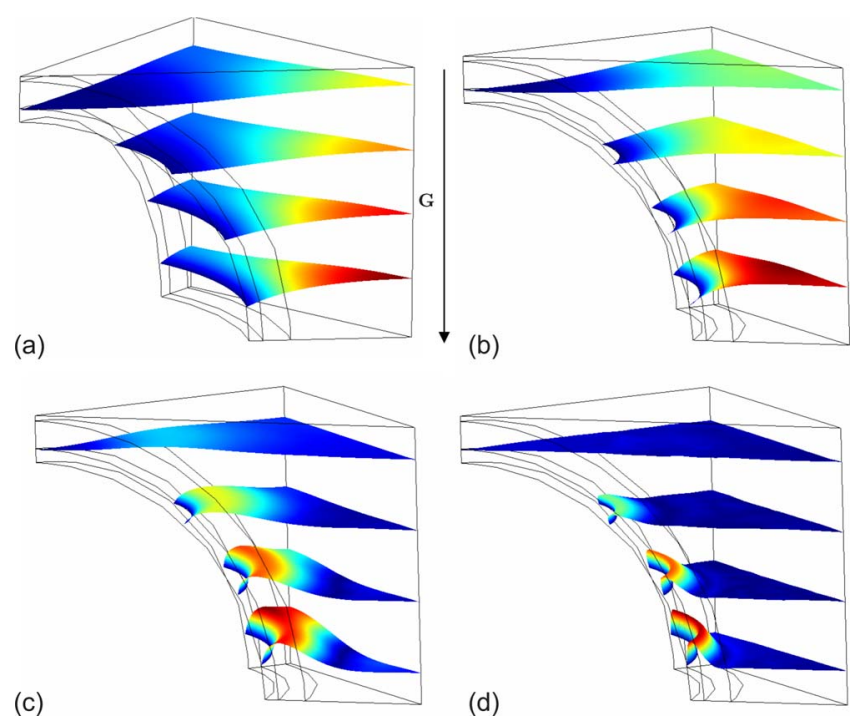

FIG. 8. (Color online) Influence of the ratio $\omega / \omega_{c}$ on the microscopic velocity field in a sc array of nonoverlapping spheres $(\phi=0.3)$ : (a) $\omega / \omega_{c}=0$, (b) $\omega / \omega_{c}=1$, (c) $\omega / \omega_{c}=10$, and (d) $\omega / \omega_{c}=100$. 

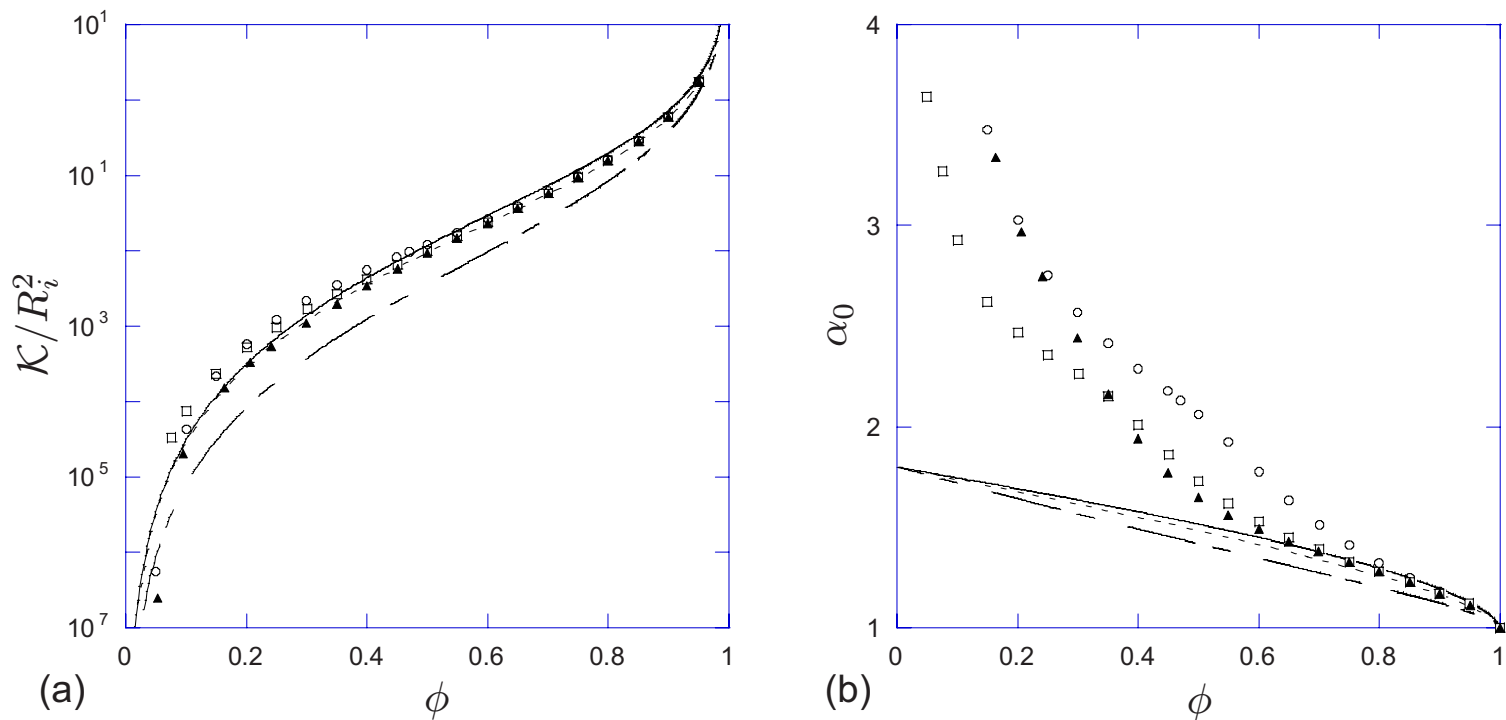

FIG. 9. (Color online) Dependence of the dimensionless permeability and the low frequency tortuosity on the porosity. Comparison between numerical results and self-consistent estimates: P-estimate (continuous line), V-estimate (mixed line), and C-estimate (dashed line). ( $\bigcirc)$, ( $\square$ ), and ( $\mathbf{\Delta})$ correspond to numerical results of sc, bcc, and fcc microstructures, respectively.

when the porosity is very small. The form factor $M$ of the three periodic microstructures presents a maximum for porosity corresponding to the maximum packing.

c. Whole frequency range. Figures 11 and 12 present the dependence of the dimensionless real and imaginary parts of $\mathbf{H}$ with the dimensionless frequency $\omega / \omega_{c}$ on porosities equal to 0.7 and 0.3 , respectively. Once again, when the porosity is large, Fig. 11 shows that $\mathrm{P}$ - and C-estimates mainly track numerical results of the bcc and fcc microstructures in the whole dimensionless frequency range. When the porosity is small, the discrepancy with estimates are more significant in the whole frequency range.

In conclusion, considering a periodic array of overlapping and nonoverlapping spheres, the three estimates are quite good qualitatively. Quantitatively, the C- and

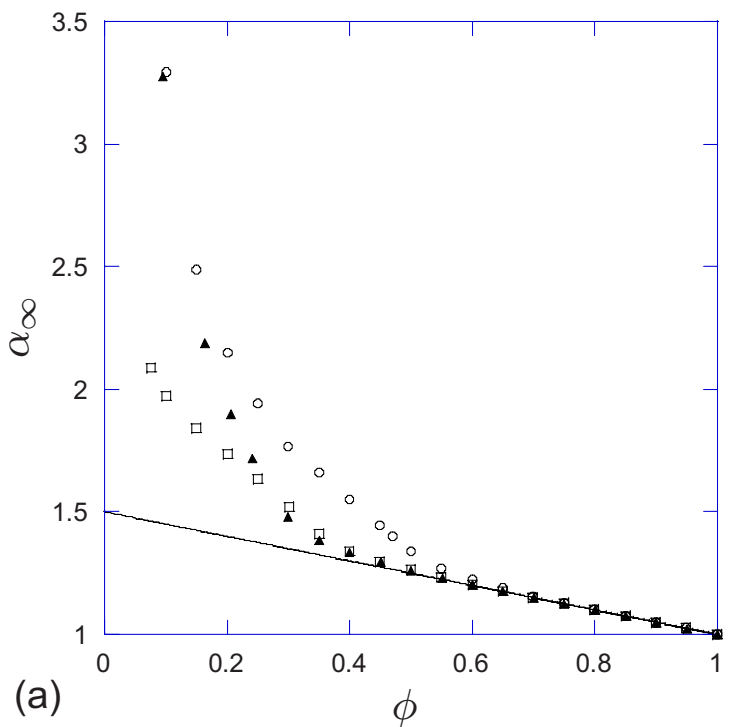

P-estimates are more accurate in the whole frequency range. This accuracy is fairly good for the four parameters $\left(K, \alpha_{0}\right.$, $\alpha_{\infty}$, and $M$ ) when the porosity is larger than 0.5 . For a lower porosity, discrepancy up to a factor of around 2 may be observed.

\section{B. Bounds of dynamic permeability for polydisperse sphere packing}

Consider granular media constituted by spherical fixed inclusions $\mathcal{I}\left\{\beta_{J}, R_{J}\right\}$ of volume $\Omega_{J}$ filling all the space, whose REV (Fig. 13) is denoted as $\hat{\Omega}=\cup \mathcal{I}\left\{\beta_{J}, R_{J}\right\}$ (index ${ }_{J}$ relates to inclusion $J$ and exponent * relates to the media),

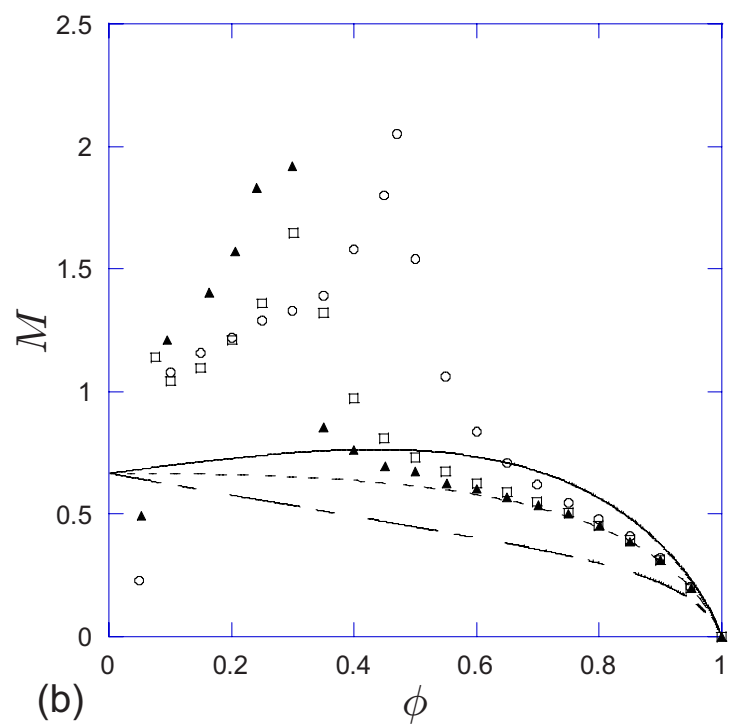

FIG. 10. (Color online) Dependence of the high frequency tortuosity and the coefficient $M$ on the porosity. Comparison between numerical results and self-consistent estimates: P-estimate (continuous line), V-estimate (mixed line), and C-estimate (dashed line). ( $\bigcirc$ ), ( $\square$ ), and $(\boldsymbol{\Lambda})$ correspond to numerical results of sc, bcc, and fcc microstructures, respectively. 


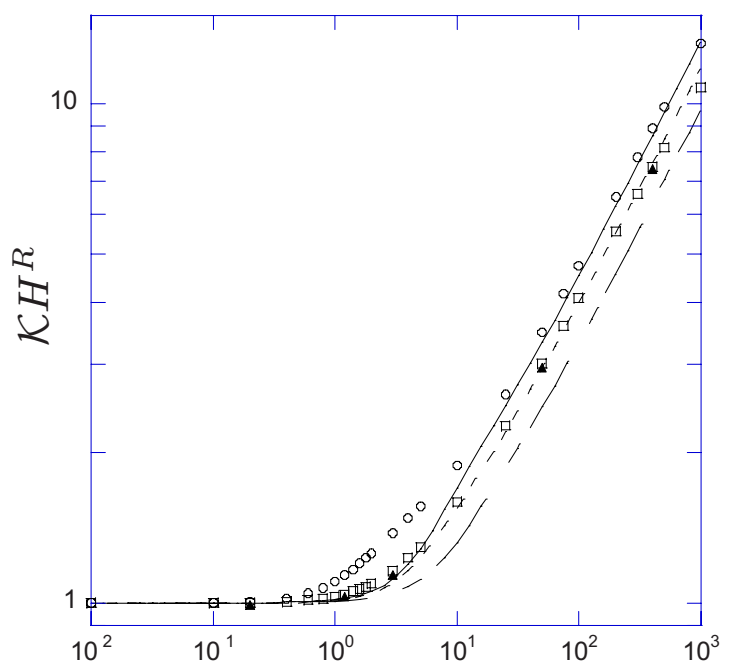

(a)

$\omega / \omega_{c}$

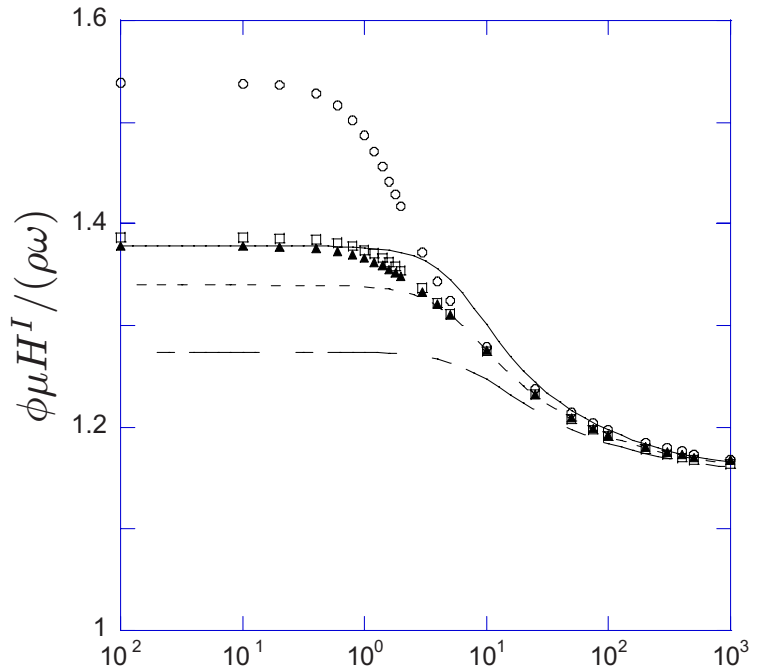

(b)

$\omega / \omega_{c}$

FIG. 11. (Color online) Comparison between numerical results and dimensionless estimates $H_{p}, H_{v}$, and $H_{c}$ vs $\omega / \omega_{c}$ when the porosity $\phi=0.7$ : P-estimate (continuous line), V-estimate (mixed line), and C-estimate (dashed line). ( $($ ), ( $\square$ ), and $(\mathbf{\Delta})$ correspond to numerical results of sc, bcc, and fcc microstructures, respectively.

$$
\hat{\Omega}=\cup \mathcal{I}_{J}\left\{\beta_{J}, R_{J}\right\}, \quad|\hat{\Omega}|=\sum_{J} \Omega_{J} .
$$

In statics, the $\mathrm{P}$ - and V-solutions enable us to set bounds to the intrinsic permeability $\mathcal{K}^{* 24}$. For the effective conduction, i.e., the tortuosity $\alpha_{\infty}^{*}$, Hashin and Shtrikman ${ }^{10}$ proved that the SCM estimates are exact values for any arrangement of inclusions of the same solid concentration $\mathcal{I}\left\{\beta, R_{J}\right\}$, filling the whole space. Using an energetic approach, these results are extended to the real and imaginary parts of the dynamic permeability in the whole frequency range.

a. Complex power function. Assume that the medium undergoes a unit macroscopic pressure gradient $\nabla P=\mathbf{e}_{\mathrm{z}}$ corresponding to a macroscopic homogeneous flux $\mathbf{V}$ $=-\left(K^{*} / \mu\right) \mathbf{e}_{\mathrm{z}}$. Among the local fields in the fluid that fulfill the Navier-Stokes equation, the incompressibility, and the adherence condition, let us distinguish ([ ] stands here for the jump through the inclusion boundary) the following.

- "stress-continuous" velocity field $\mathbf{v}_{p}$ such that on any inclusion boundary, $\left[\boldsymbol{\sigma}_{p} \mathbf{n}\right]=0$,

- velocity-continuous fields $\mathbf{v}_{v}$ such that on any inclusion boundary, $\left[\mathbf{v}_{v}\right]=0$, and

- the exact solution, $\mathbf{v}^{*}$, which is the only field whose stress and velocity are continuous.

Introduce for any stress- and velocity-continuous fields defined on the whole inclusions of the REV the following complex power function $E$ :

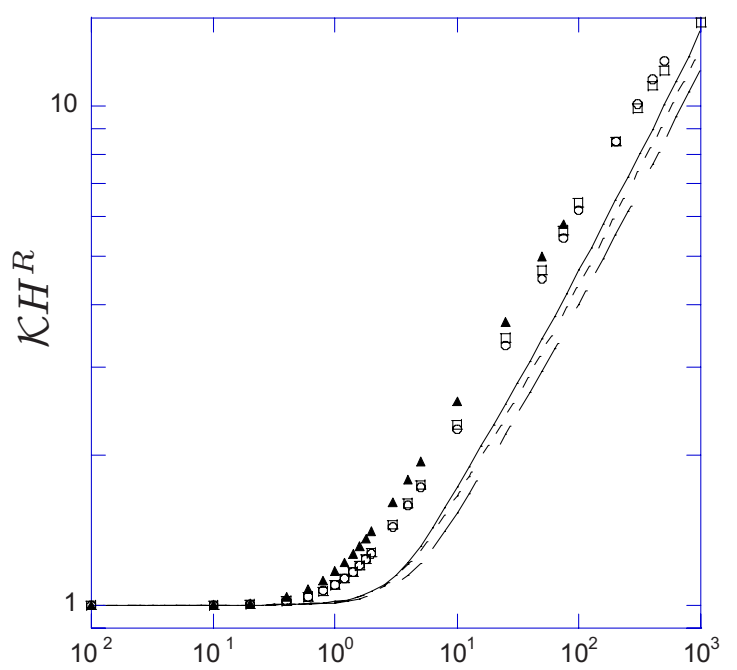

(a)

$$
\omega / \omega_{c}
$$

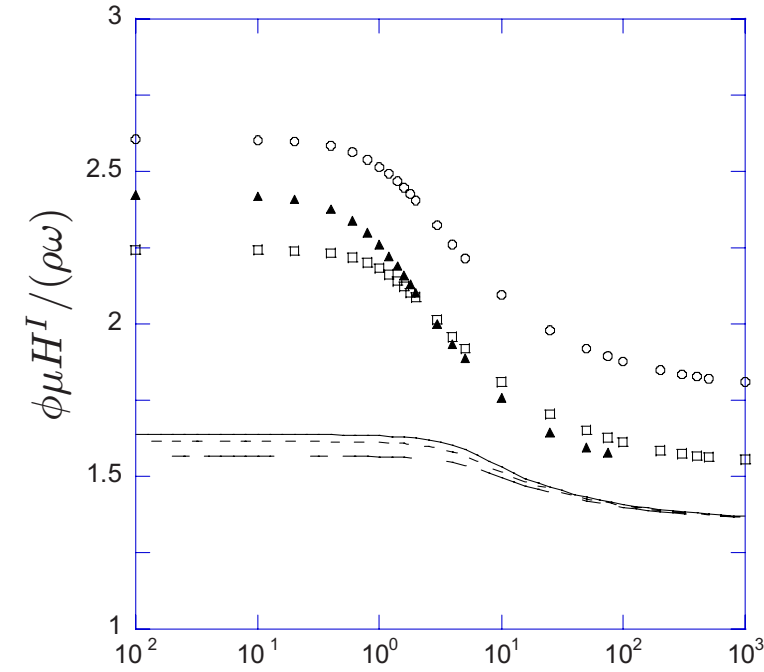

(b)

FIG. 12. (Color online) Comparison between numerical results and dimensionless estimates $H_{p}, H_{v}$, and $H_{c}$ vs $\omega / \omega_{c}$ when the porosity $\phi=0.3$ : P-estimate (continuous line), V-estimate (mixed line), and C-estimate (dashed line). ( $\bigcirc),(\square)$, and ( $\mathbf{\Delta}$ ) correspond to numerical results of sc, bcc, and fcc microstructures, respectively. 


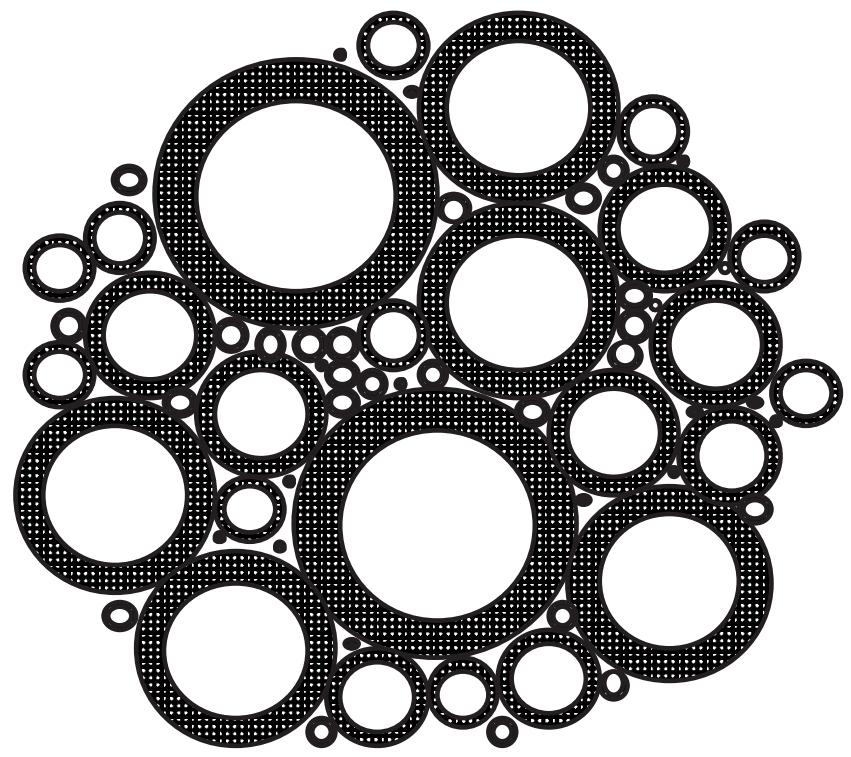

FIG. 13. REV of granular media constituted by spherical fixed inclusions $\mathcal{I}\left\{\beta_{J}, R_{J}\right\}$ of volume $\Omega_{J}$ filling all the space.

$$
\begin{aligned}
2 E\left(\mathbf{v}_{p}, \mathbf{v}_{v}\right)= & \sum_{J}\left\{2 \mu \int_{\Omega_{f J}} \mathbf{D}\left(\mathbf{v}_{p}-\mathbf{v}_{v}\right): \overline{\mathbf{D}\left(\mathbf{v}_{p}-\mathbf{v}_{v}\right)} d \Omega\right. \\
& \left.+i \omega \rho \int_{\Omega_{f J}}\left(\mathbf{v}_{p}-\mathbf{v}_{v}\right) \cdot \overline{\left(\mathbf{v}_{p}-\mathbf{v}_{v}\right)} d \Omega\right\} .
\end{aligned}
$$

By construction, the real and imaginary parts of $E$ are positive, and their minima (zero) are reached when $\mathbf{v}_{p}=\mathbf{v}_{v}=\mathbf{v}^{*}$. Developing $E$ gives

$$
\begin{aligned}
2 E\left(\mathbf{v}_{p}, \mathbf{v}_{v}\right)= & \sum_{J}\left\{\int_{\Omega_{f J}}\left[2 \mu \mathbf{D}\left(\mathbf{v}_{p}\right): \overline{\mathbf{D}\left(\mathbf{v}_{p}\right)}+i \omega \rho \mathbf{v}_{p} \cdot \overline{\mathbf{v}_{p}}\right] d \Omega\right. \\
& +\int_{\Omega_{f J}}\left[2 \mu \mathbf{D}\left(\mathbf{v}_{v}\right): \overline{\mathbf{D}\left(\mathbf{v}_{v}\right)}+i \omega \rho \mathbf{v}_{v} \cdot \overline{\mathbf{v}_{v}}\right] d \Omega \\
& -\int_{\Omega_{f J}}\left[2 \mu \mathbf{D}\left(\mathbf{v}_{p}\right): \overline{\mathbf{D}\left(\mathbf{v}_{v}\right)}+i \omega \rho \mathbf{v}_{p} \cdot \overline{\mathbf{v}_{v}}\right] d \Omega \\
& \left.-\int_{\Omega_{f J}}\left[2 \mu \overline{\mathbf{D}\left(\mathbf{v}_{p}\right)}: \mathbf{D}\left(\mathbf{v}_{v}\right)+i \omega \rho \mathbf{v}_{p} \cdot \mathbf{v}_{v}\right] d \Omega\right\} .
\end{aligned}
$$

Integrating by part and using the properties of the fields $\left(\nabla \cdot \mathbf{v}_{v}=0 ; \nabla \cdot \boldsymbol{\sigma}_{p}=0\right)$, the two last integrals may be transformed into surface integrals, so that

$$
\begin{aligned}
2 E\left(\mathbf{v}_{p}, \mathbf{v}_{v}\right)= & 2 E\left(\mathbf{v}_{p}, \mathbf{0}\right)+2 E\left(\mathbf{0}, \mathbf{v}_{v}\right) \\
& -\sum_{J} \int_{\partial \Omega_{J}}\left[\left(\sigma_{p} \cdot \mathbf{n}\right) \cdot \overline{\mathbf{v}}_{v}+\left(\bar{\sigma}_{p} \cdot \mathbf{n}\right) \cdot \mathbf{v}_{v}\right] d s .
\end{aligned}
$$

b. Bound of the real part of $K^{*}$. A stress-continuous field $\widetilde{\mathbf{v}}_{p}$ can be built by partitioning the media inclusion by inclusion, and setting in each $\mathcal{I}_{J}, \widetilde{\mathbf{v}}_{p}=\varpi_{p J}$, where $\varpi_{p}$ was defined in Sec. III B 1. Consequently, the condition $\widetilde{\sigma}_{p} \cdot \mathbf{n}=P \cdot \mathbf{n}$ is satisfied on the boundary of each inclusion. Now, choose for velocity-continuous field $\mathbf{v}_{v}$ the solution $\mathbf{v}^{*}$. Then it comes successively: (i) By construction of the fields $\varpi_{p J}$ [see Eq. (39)],

$$
\begin{aligned}
& \int_{\Omega_{f J}} {\left[2 \mu \mathbf{D}_{y}\left(\overline{\varpi_{p J}}\right): \mathbf{D}_{y}\left(\overline{\varpi_{p J}}\right)+i \omega \rho \varpi_{p J} \cdot \overline{\varpi_{p J}}\right] d \Omega } \\
&=-\nabla P \cdot \int_{\Omega_{f J}} \overline{\varpi_{p J}} d \Omega=\Omega_{J} \frac{\overline{K_{p J}}}{\mu},
\end{aligned}
$$

so that

$$
2 E\left(\widetilde{\mathbf{v}}_{p}, \mathbf{0}\right)=\sum_{J} \Omega_{J} \frac{\overline{K_{p J}}}{\mu} .
$$

(ii) Because of the energy consistency of the dynamic permeability $K^{*}$ [see Eq. (16)],

$$
\begin{aligned}
2 E\left(\mathbf{0}, \mathbf{v}^{*}\right) & =\sum_{J} \int_{\partial \Omega_{J}}\left[2 \mu \mathbf{D}_{y}\left(\mathbf{v}^{*}\right): \mathbf{D}_{y}\left(\overline{\mathbf{v}^{*}}\right)+i \omega \rho \mathbf{v}^{*} \cdot \overline{\mathbf{v}^{*}}\right] \\
& =-\hat{\Omega} \nabla_{x} P \cdot \overline{\mathbf{v}^{*}}=\hat{\Omega} \frac{\overline{K^{*}}}{\mu} .
\end{aligned}
$$

(iii) Finally, using the continuity of the stress induced by $\varpi_{p J}$ on $\partial \Omega_{J}$ with the macroscopic pressure,

$$
\begin{aligned}
& -\sum_{J} \int_{\partial \Omega_{J}}\left[\left(\widetilde{\sigma}_{p} \cdot \mathbf{n}\right) \cdot \overline{\mathbf{v}}^{*}+\left(\widetilde{\sigma}_{p} \cdot \mathbf{n}\right) \cdot \mathbf{v}^{*}\right] d s \\
& =\sum_{J} \int_{\partial \Omega_{J}}\left[P \mathbf{n} \cdot \overline{\mathbf{v}}^{*}+\bar{P} \mathbf{n} \cdot \mathbf{v}^{*}\right] d s \\
& =\sum_{J} \int_{\Omega_{f J}}\left[\nabla P \overline{\mathbf{v}}^{*}+\nabla \bar{P} \cdot \mathbf{v}^{*}\right] d s \\
& =\hat{\Omega}[\nabla P \cdot \overline{\mathbf{V}}+\nabla \bar{P} \cdot \mathbf{V}]=-2 \hat{\Omega} \frac{K^{* R}}{\mu} .
\end{aligned}
$$

Reporting Eqs. (55)-(57) into Eq. (54) yields

$$
\begin{aligned}
2 E\left(\widetilde{\mathbf{v}}_{p}, \mathbf{v}^{*}\right) & =\sum_{J}\left\{\Omega_{J} \frac{\overline{K_{p J}}}{\mu}+\Omega_{J} \frac{\overline{K^{*}}}{\mu}\right\}-2 \hat{\Omega} \frac{K^{* R}}{m} \\
& =\hat{\Omega}\left\{\sum_{J} \frac{\Omega_{J}}{\hat{\Omega}} \frac{\overline{K_{p J}}}{\mu}-\frac{K^{*}}{\mu}\right\},
\end{aligned}
$$

and from the positiveness of the real part of $E\left(\widetilde{\mathbf{v}}_{p}, \mathbf{v}^{*}\right)$ one deduces the inequality, giving an upper bound of $K^{* R}$,

$$
\left\{\sum_{J} \frac{\Omega_{J}}{\hat{\Omega}} K_{p, J}^{R}-K^{* R}\right\} \geqslant 0 .
$$

c. Bound of the imaginary part of $K^{*}$. Similarly, choosing $\mathbf{v}_{v}=i \mathbf{v}^{*}$ leads us to consider $2 E\left(\widetilde{\mathbf{v}}_{p}, i \mathbf{v}^{*}\right)$. Observing that $E\left(\mathbf{0}, i \mathbf{v}^{*}\right)=E\left(\mathbf{0}, \mathbf{v}^{*}\right)$ and that

$$
\begin{gathered}
-\sum_{J} \int_{\partial \Omega_{J}}\left[\left(\widetilde{\sigma}_{p} \cdot \mathbf{n}\right) \cdot \overline{i \mathbf{V}^{*}}+\left(\overline{\widetilde{\sigma}_{p}} \cdot \mathbf{n}\right) \cdot i \mathbf{V}^{*}\right] d s \\
=\hat{\Omega}[\nabla P \cdot \overline{i \mathbf{V}}+\nabla \overline{\mathbf{P}} \cdot i \mathbf{V}]=+2 i \hat{\Omega} \frac{K^{* I}}{\mu},
\end{gathered}
$$

we obtain 


$$
\begin{aligned}
2 E\left(\widetilde{\mathbf{v}}_{p}, i \mathbf{v}^{*}\right) & =\sum_{J}\left\{\Omega_{J} \frac{\overline{K_{p J}}}{\mu}+\Omega_{J} \frac{\overline{K^{*}}}{\mu}\right\}+2 i \hat{\Omega} K^{* I} \\
& =\hat{\Omega}\left\{\sum_{J} \frac{\Omega_{J}}{\hat{\Omega}} \frac{\overline{K_{p J}}}{\mu}+\frac{K^{*}}{\mu}\right\} .
\end{aligned}
$$

Expressing the positiveness of the imaginary part of $E\left(\widetilde{\mathbf{v}}_{p}, i \mathbf{v}^{*}\right)$ (recall that $\left.K^{* I}<0\right)$,

$$
\left\{\sum_{J} \frac{\Omega_{J}}{\hat{\Omega}}\left|K_{p J}^{I}\right|-\left|K^{* I}\right|\right\} \geqslant 0,
$$

which yields to upper bounds of $\left|K^{* I}\right|$.

d. Bound of the imaginary parts of $K^{*}$ and $H^{*}$. A similar approach based on field $\varpi_{v J}$ leads to upper bounds of $H^{* R}$ and $H^{* I}$ (see Appendix B). Finally, the bounds valid in the whole frequency range read

$$
\begin{aligned}
& K^{R *} \leqslant \sum_{J} \frac{\Omega_{J}}{\hat{\Omega}} K_{p J}^{R}, \quad\left|K^{I *}\right| \leqslant \sum_{J} \frac{\Omega_{J}}{\hat{\Omega}}\left|K_{p J}^{I}\right|, \\
& H^{R *} \leqslant \sum_{J} \frac{\Omega_{J}}{\hat{\Omega}} H_{v J}^{R}, \quad H^{I *} \leqslant \sum_{J} \frac{\Omega_{J}}{\hat{\Omega}} H_{v J}^{I} .
\end{aligned}
$$

These bounds are valid for any random or periodic spatial distribution of inclusions. In the low and high frequency limits, these general bounds give

$$
\begin{aligned}
& {\left[\sum_{J} \frac{\Omega_{J}}{\hat{\Omega}} \frac{1}{\mathcal{K}_{v J}}\right]^{-1} \leqslant \mathcal{K}^{*} \leqslant \sum_{J} \frac{\Omega_{J}}{\hat{\Omega}} \mathcal{K}_{p J},} \\
& \frac{\alpha_{0}^{*}}{\phi^{*}} \leqslant \sum_{J} \frac{\Omega_{J}}{\hat{\Omega}} \frac{\alpha_{0 v J}}{\phi_{J}}, \\
& {\left[\sum_{J} \frac{\Omega_{J}}{\hat{\Omega}} \frac{\phi_{J}}{a_{\infty}}\right]^{-1} \leqslant \frac{\alpha_{\infty}^{*}}{\phi^{*}} \leqslant \sum_{J} \frac{\Omega_{J}}{\hat{\Omega}} \frac{\alpha_{\infty J}}{\phi_{J}} .}
\end{aligned}
$$

Note that if all the inclusions present the same porosity $\left(\phi_{J}\right.$ $=\phi^{*}$ ), the upper and lower bounds are identical for the tortuosity at high frequency, so that the estimates provide the exact value $\left(\alpha_{\infty}^{*}=\alpha_{\infty J}\right)$, in agreement with Hashin's result.

\section{CONCLUSION}

This study provides three analytical expressions of the dynamic permeability of granular media and emphasizes the physical assumption sustaining these results. Theses estimates follow the physical requirements in the whole frequency range and the whole porosity range. Among the two estimates energetically consistent, the P-estimate gives a good qualitative approximation of numerical results computed by the finite element method for periodic arrays (sc, bcc, and fcc) of overlapping and nonoverlapping spheres. Quantitatively, when $\phi>0.5$, the agreement is excellent and is still reasonable otherwise. Although the zero vorticity assumption is not energetically consistent, the associated estimate leads to a value very close to the consistent P-estimate (and even more accurate as for periodic arrays of spheres). The P- and V-estimate enable us to bound the dynamic permeability of polydisperse sphere packing.

Meanwhile the spherical fluid-solid inclusion is an extremely simple and regular 3D pore geometry, these results underline the great sensitivity of the macroscopic parameters to the morphology of the medium. Nevertheless such a geometry is not sufficient to capture all the complexities of the flow in actual geometry, such as that of overlapping spheres. For this reason, the present estimates may apply to porous media whose pores are sufficiently regular (for instance, granular media made of polyhedron packing instead of sphere packing) but should be used cautiously otherwise.

It is worth mentioning that the rigorous physical analysis performed with the HPM plays a determining role in establishing these estimates. In the problem under scrutiny, other results would have been obtained if, instead of the dynamic Darcy law, a macroscopic Brinkman law was assumedwhich actually occurs for a very dilute array of particles $^{25}$ - or a macroscopic viscoelastic behavior, which actually occurs for fluid of very high viscosity. ${ }^{26}$ Note finally that the obtained results may easily be extended to linear viscoelastic saturating fluids.

\section{APPENDIX A: VARIATION OF REAL AND IMAGINARY PARTS OF $H$ VERSUS FREQUENCY}

The leading idea to establish the variations of $H$ (assumed to be isotropic, or in a given principal direction) is to calculate the virtual crossed energies of a particular velocity field - solution at a given frequency — under a particular velocity field-solution at another frequency-and reciprocally. For this purpose, let us introduce the field solutions $\mathbf{u}$ and $\mathbf{u}^{\prime}$ corresponding to unit real mean velocity at the frequency $\omega$ (respectively $\omega^{\prime}$ ), i.e.,

$$
\langle\mathbf{u}\rangle=\left\langle\mathbf{u}^{\prime}\right\rangle=\mathbf{e}, \quad|\mathbf{e}|=1, \quad \arg \mathbf{e}=0 .
$$

The associated forcing pressure gradients are $-\mu H \mathbf{e}$ and $-\mu H^{\prime} \mathbf{e}$, and the corresponding variational formulation [Eq. (15)] reads, for $\mathbf{u}$ [and similarly for $\left.\mathbf{u}^{\prime}, \omega^{\prime}, H^{\prime}=H\left(\omega^{\prime}\right)\right]$,

$$
\forall \mathbf{w} \in \mathcal{W},
$$

$$
2 \mu\left\langle\mathbf{D}_{y}(\mathbf{u}): \mathbf{D}_{y}(\mathbf{w})\right\rangle+i \omega \rho\langle\mathbf{u} \cdot \mathbf{w}\rangle=+\mu H \mathbf{e}\langle\mathbf{w}\rangle .
$$

Choose now in the $\omega$-variational formulation (or $\omega^{\prime}$ ), the field test $\mathbf{w}=H^{\prime} \mathbf{u}^{\prime}$ (or $\left.\mathbf{w}=H \mathbf{u}\right)$. One obtains

$$
\begin{aligned}
& 2 \mu\left\langle\mathbf{D}_{y}(\mathbf{u}): \mathbf{D}_{y}\left(\mathbf{u}^{\prime}\right)\right\rangle H^{\prime}+i \omega \rho\left\langle\mathbf{u} \cdot \mathbf{u}^{\prime}\right\rangle H^{\prime}=\mu H H^{\prime}, \\
& 2 \mu\left\langle\mathbf{D}_{y}\left(\mathbf{u}^{\prime}\right): \mathbf{D}_{y}(\mathbf{u})\right\rangle H+i \omega^{\prime} \rho\left\langle\mathbf{u}^{\prime} \cdot \mathbf{u}\right\rangle H=\mu H H^{\prime} .
\end{aligned}
$$

Subtracting these two expressions yields to the following identity, valid for any frequency $\omega$ and $\omega^{\prime}$ :

$$
2 \mu\left\langle\mathbf{D}_{y}(\mathbf{u}): \mathbf{D}_{y}\left(\mathbf{u}^{\prime}\right)\right\rangle\left(H^{\prime}-H\right)+i \rho\left\langle\mathbf{u} \cdot \mathbf{u}^{\prime}\right\rangle\left(\omega H^{\prime}-\omega^{\prime} H\right)=0 .
$$

Taking $\omega$ close to $\omega^{\prime}$, one deduces 


$$
2 \mu\left\langle\mathbf{D}_{y}(\mathbf{u}): \mathbf{D}_{y}(\mathbf{u})\right\rangle \frac{d H}{d \omega}+i \rho\langle\mathbf{u} \cdot \mathbf{u}\rangle \omega^{2} \frac{d(H / \omega)}{d \omega}=0 .
$$

However, choosing $\mathbf{w}=\mathbf{u}$ in the $\omega$-variational formulation gives

$$
2 \mu\left\langle\mathbf{D}_{y}(\mathbf{u}): \mathbf{D}_{y}(\mathbf{u})\right\rangle+i \rho\langle\mathbf{u} \cdot \mathbf{u}\rangle \omega=\mu H .
$$

Then from Eqs. (60) and (61), we get

$$
\frac{d H}{d \omega}=\frac{i \rho}{\mu}\langle\mathbf{u} \cdot \mathbf{u}\rangle, \quad \omega^{2} \frac{d(H / \omega)}{d \omega}=-2\left\langle\mathbf{D}_{y}(\mathbf{u}): \mathbf{D}_{y}(\mathbf{u})\right\rangle,
$$

and coming back to the real and imaginary parts,

$$
\begin{aligned}
& 2 \frac{d H^{R}}{d \omega}=\frac{i \rho}{\mu}\langle(\mathbf{u}+\overline{\mathbf{u}}) \cdot(\mathbf{u}-\overline{\mathbf{u}})\rangle, \\
& 2 i \omega^{2} \frac{d\left(H^{I} / \omega\right)}{d \omega}=-2\left\langle\left[\mathbf{D}_{y}(\mathbf{u})+\mathbf{D}_{y}(\overline{\mathbf{u}})\right]:\left[\mathbf{D}_{y}(\mathbf{u})-\mathbf{D}_{y}(\overline{\mathbf{u}})\right]\right\rangle .
\end{aligned}
$$

In order to establish the sign of these derivatives, we use $\mathbf{w}=\mathbf{u}-\overline{\mathbf{u}}$ as a field test in the natural and conjugate $\omega$-variational formulations. One obtains

$$
\begin{aligned}
& 2 \mu\left\langle\mathbf{D}_{y}(\mathbf{u}):\left[\mathbf{D}_{y}(\mathbf{u})-\mathbf{D}_{y}(\overline{\mathbf{u}})\right]\right\rangle+i \omega \rho\langle\mathbf{u} \cdot(\mathbf{u}-\overline{\mathbf{u}})\rangle=0, \\
& 2 \mu\left\langle\mathbf{D}_{y}(\overline{\mathbf{u}}):\left[\mathbf{D}_{y}(\mathbf{u})-\mathbf{D}_{y}(\overline{\mathbf{u}})\right]\right\rangle-i \omega \rho\langle\overline{\mathbf{u}} \cdot(\mathbf{u}-\overline{\mathbf{u}})\rangle=0,
\end{aligned}
$$

which yields the following by summing and subtracting:

$$
\begin{aligned}
& i \omega \rho\langle(\mathbf{u}+\overline{\mathbf{u}}) \cdot(\mathbf{u}-\overline{\mathbf{u}})\rangle=8 \mu\left\langle\left[\mathbf{D}_{y}(\mathbf{u})\right]^{I}:\left[\mathbf{D}_{y}(\mathbf{u})\right]^{I}\right\rangle, \\
& \mu\left\langle\left[\mathbf{D}_{y}(\mathbf{u})+\mathbf{D}_{y}(\overline{\mathbf{u}})\right]:\left[\mathbf{D}_{y}(\mathbf{u})-\mathbf{D}_{y}(\overline{\mathbf{u}})\right]\right\rangle=8 i \omega \rho\left\langle\left(\mathbf{u}^{I}\right)^{2}\right\rangle .
\end{aligned}
$$

In the left hand side terms, the squares of the imaginary parts are positive, and therefore,

$$
\frac{d H^{R}}{d \omega} \geqslant 0, \quad \frac{d\left(H^{I} / \omega\right)}{d \omega} \leqslant 0,
$$

which proves the monotonic increases in the viscous part with the frequency and, conversely, the monotonic decrease in tortuosity (or added mass) in the whole frequency range.

\section{APPENDIX B: BOUNDS OF REAL AND IMAGINARY PARTS OF $\boldsymbol{H}^{*}$}

We assume now in $\hat{\Omega}$ a macroscopic flux $\mathbf{V}=-\mathbf{e}_{\mathbf{z}}$, which corresponds to the macroscopic pressure gradient $\nabla P$ $=\mu H^{*} \mathbf{e}_{\mathbf{z}}$ and the exact flow $\mathbf{v}^{*} H^{*}$. A velocity-continuous field $\widetilde{\mathbf{v}}_{v}$ is built by setting in each $\mathcal{I}_{J}, \widetilde{\mathbf{v}}_{v}=\varpi_{v J} H_{v J}$, where $\varpi_{v}$ is defined in Sec. III B 2.

Let us calculate $2 E\left(\mathbf{v}^{*} H^{*}, \widetilde{\mathbf{v}}_{v}\right)$. On the boundary of each inclusion, $\tilde{\mathbf{v}_{v}} \cdot \mathbf{n}=\mathbf{v}$, and $\mathbf{v}^{*} H^{*}$ is chosen as the stresscontinuous field,

$$
\begin{aligned}
& \left.-\sum_{J} \int_{\partial \Omega_{J}}\left[\left(H^{*} \sigma^{*} \cdot \mathbf{n}\right) \cdot \overline{\widetilde{\mathbf{v}}_{v}}+\overline{\left(H^{*} \sigma^{*}\right.} \cdot \mathbf{n}\right) \cdot \widetilde{\mathbf{v}}_{v}\right] d s \\
& \left.\quad=-\sum_{J} \int_{\partial \Omega_{f}}\left[\left(H^{*} \sigma^{*} \cdot \mathbf{n}\right) \cdot \overline{\mathbf{V}}+\overline{\left(H^{*} \sigma^{*}\right.} \cdot \mathbf{n}\right) \cdot \mathbf{V}\right] d s \\
& \quad=\sum_{J} \int_{\partial \Omega_{f}}(P \mathbf{n} \cdot \overline{\mathbf{V}}+\bar{P} \mathbf{n} \cdot \mathbf{V}) d s
\end{aligned}
$$

$$
=\hat{\Omega}[\nabla P \cdot \overline{\mathbf{V}}+\nabla \bar{P} \cdot \mathbf{V}]=-2 \hat{\Omega} H^{* R} .
$$

Then, from (i) the above equality, (ii) Eq. (16) applied to $\mathbf{v}^{*} H^{*}$, and (iii) Eq. (39) applied to $\varpi_{v J} H_{v J}$, we get,

$$
\begin{aligned}
2 E\left(\mathbf{v}^{*} H^{*}, \tilde{\mathbf{v}_{v}}\right) & =\sum_{J}\left\{\Omega_{J} H^{*}+\Omega_{J}\left(H_{v J} \overline{H_{v J}}\right) \overline{K_{v J}}\right\}-2 \hat{\Omega} H^{* R} \\
& =\hat{\Omega}\left\{\sum_{J} \frac{\Omega_{J}}{\hat{\Omega}} H_{v J}-\overline{H^{*}}\right\} .
\end{aligned}
$$

Furthermore, one may also establish

$$
\begin{aligned}
2 E\left(-i \mathbf{v}^{*} H^{*}, \widetilde{\mathbf{v}}_{v}\right)= & \sum_{J}\left\{\Omega_{J} H^{*}+\Omega_{J}\left(H_{v J} \overline{H_{v J}}\right) \overline{K_{v J}}\right\} \\
& -2 \hat{\Omega} i H^{* I}=\hat{\Omega}\left\{\sum_{J} \frac{\Omega_{J}}{\hat{\Omega}} H_{v J}+\overline{H^{*}}\right\} .
\end{aligned}
$$

The positiveness of the real part of $E\left(\mathbf{v}^{*} \overline{H^{*}}, \tilde{\mathbf{v}_{v}}\right)$ and of the imaginary part of $E\left(-i \mathbf{v}^{*} \overline{H^{*}}, \widetilde{\mathbf{v}_{v}}\right)$ gives

$$
H^{* R}<\sum_{J} \frac{\Omega_{J}}{\hat{\Omega}} H_{v J}^{R}, \quad H^{* I}<\sum_{J} \frac{\Omega_{J}}{\hat{\Omega}} H_{v J}^{I} .
$$

${ }^{1}$ M. A. Biot, "Theory of propagation of elastic waves in a fluid saturated porous solid. I. low frequency range. II. Higher frequency range," J. Acoust. Soc. Am. 28, 168-191 (1956).

${ }^{2} \mathrm{~K}$. Attenborough, "Acoustical characteristics of rigid fibrous absorbents and granular Media,” J. Acoust. Soc. Am. 73, 785-799 (1983).

${ }^{3}$ D. L. Johnson, J. Koplik, and R. Dashen, "Theory of dynamic permeability and tortuosity in fluid saturated porous media," J. Fluid Mech. 176, 379-402 (1987).

${ }^{4}$ J.-F. Allard, Propagation of Sound in Porous Media (Elsevier Applied Science, England, 1993).

${ }^{5}$ J.-L. Auriault, "Dynamic behaviour of a porous medium saturated by a Newtonian fluid,” Int. J. Eng. Sci. 18, 775-795 (1980).

${ }^{6}$ J.-L. Auriault and E. Sanchez-Palencia, "Etude du comportement macroscopique d'un milieu poreux saturé déformable (Study of the macrocopic behaviour of saturated deformable porous media)," Journal de Mécanique 16, 576-603 (1977).

${ }^{7}$ E. Sanchez-Palencia, "Non-homogeneous media and vibration theory," Lecture Notes in Physics (Springer-Verlag, Berlin, 1980), Vol. 127.

${ }^{8}$ A. S. Sangani and A. Acrivos, "Slow flow past periodic periodic arrays of cylinders with application to heat transfer," Int. J. Multiphase Flow 8, 193-206 (1982).

${ }^{9}$ A. L. Berdichevsky and Z. Cai, "Perform permeability predictions by self consistent method and finite element simulation," Polym. Compos. 14, 132-143 (1993).

${ }^{10} \mathrm{C}$. Boutin, "Study of permeability by periodic and self consistent homogenisation," Eur. J. Mech. A/Solids 19, 603-632 (2000).

${ }^{11} \mathrm{~V}$. Tarnow, "Air flow resistivity of models of fibrous acoustic materials," J. Acoust. Soc. Am. 100, 3706-3713 (1996).

${ }^{12}$ J.-L. Auriault, L. Borne, and R. Chambon, "Dynamics of porous saturated media, checking of the generalized law of Darcy," J. Acoust. Soc. Am. 77, 1641-1650 (1985).

${ }^{13}$ M.-Y. Zhou and P. Sheng, "First principle calculations of dynamic permeability in porous media," Phys. Rev. B 39, 12027-12039 (1989).

${ }^{14}$ A. M. Chapman and J. J. L. Higdon, "Oscillatory stokes flow a in periodic porous media,” Phys. Fluids A 4, 2099-2116 (1992).

${ }^{15} \mathrm{O}$. Umnova, K. Attenborough, and K. Ming Li, "Cell model calculations of dynamic drag parameters in packings of spheres," J. Acoust. Soc. Am. 107, 3113-3118 (2000).

${ }^{16}$ R. M. Christensen and K. H. Lo, "Solutions for effective shear properties in three phase sphere and cylinder model," J. Mech. Phys. Solids 27, 315-330 (1979).

${ }^{17}$ J.-L. Auriault, "Heterogeneous medium: Is an equivalent description possible?," Int. J. Eng. Sci. 29, 785-795 (1991).

${ }^{18}$ T. Levy, "Propagation of waves in fluid saturated porous elastic solid," Int. J. Eng. Sci. 17, 1005-1014 (1979). 
${ }^{19}$ Z. Hashin, "Assessment of self consistent scheme approximation: Conductivity of particulate composites," J. Compos. Mater. 2, 284-304 (1968).

${ }^{20}$ J.-P. Boelher, Applications of Tensor Functions in Solid Mechanics, CISM Courses and Lectures (Springer, Wien, 1987).

${ }^{21}$ I. D. Howells, "Drag due to the motion of a Newtonian fluid through a sparse random array of small fixed rigid objects," J. Fluid Mech. 64, 449-485 (1974).

${ }^{22}$ S. R. Pride, F. D. Morgan, and A. F. Gangi, "Drag force of porous medium acoustics," Phys. Rev. B 47, 4964-4978 (1993).

${ }^{23}$ COMSOL MULTIPHYSICS, Version 3.4, Reference Manual, http:// www.comsol.com (Last viewed December 1, 2007).

${ }^{24}$ Z. Hashin and S. Shtrikman, "A variational approach to the theory of elastic behavior of multiphase materials," J. Mech. Phys. Solids 11, 127140 (1963).

${ }^{25}$ T. Levy, "Fluid flow through an array of fixed particles," Int. J. Eng. Sci. 21, 11-23 (1983).

${ }^{26}$ C. Boutin and J.-L. Auriault, "Dynamic behaviour of porous media saturated by a viscoelastic fluid: Application to bituminous concretes," Int. J. Eng. Sci. 28, 1157-1181 (1990). 